\title{
ATLAS Status and Latest Results
}

\section{Carla Sbarra*}

on behalf of the ATLAS Collaboration

INFN and University of Bologna, Italy

E-mail: carla.sbarra@cern.ch

\begin{abstract}
ATLAS is a general-purpose detector located at one of the four interaction points of the Large Hadron Collider at the CERN laboratory near Geneva, Switzerland. In 2010 LHC collided proton beams at the unprecedented centre-of-mass energy of $7 \mathrm{TeV}$. The last month of operations was dedicated to $\mathrm{Pb}$-ion collisions at a centre-of-mass energy of $2.76 \mathrm{TeV}$ per nucleon pair. A survey of the status of the ATLAS detector in both runs is presented in this talk. The main results in proton-proton collisions, including the study of minimum bias events, jets, $W$ and $Z$ gauge boson production, top quark production and first searches for new physics are reviewed. The first results from heavy-ion collisions are focussed on the observation of jet quenching and studies of $J / \psi$ production as a function of event centrality.
\end{abstract}

XLIX International Winter Meeting on Nuclear Physics

24-28 January 2011

BORMIO, Italy

${ }^{*}$ Speaker. 


\section{LHC Running in 2010}

The Large Hadron Collider (LHC), the largest particle accelerator in the world, is located beneath the border between France and Switzerland, in the underground tunnel which used to host the former Large Electron-Positron (LEP) Collider. LHC has two different modes of operation: one is as proton collider with a design centre-of-mass energy of $14 \mathrm{TeV}$, the other is as $\mathrm{Pb}$-ion collider, accelerating fully ionized lead atoms. For lead ions, the design centre-of-mass energy is $2.76 \mathrm{TeV} /$ nucleon.

The rate, $R$, of events provided by accelerators is proportional to the machine instantaneous luminosity, $L$, and the event total cross section, $\sigma: R=\sigma \times L$. LHC is expected to reach a luminosity $L=2 \times 10^{33} \mathrm{~cm}^{-2} \mathrm{~s}^{-1}$ during the first years of operations with protons, while the design luminosity $L=10^{34} \mathrm{~cm}^{-2} \mathrm{~s}^{-1}$ should be reached later. The design luminosity for lead-ion beams is $\sim 10^{27} \mathrm{~cm}^{-2} \mathrm{~s}^{-1}$, substantially lower than the former because the large ion charge makes it more difficult to control beam-beam interactions. A large number of particles are produced when complex nuclei collide, and the inelastic cross section is a strong function of the atomic number A: $\sigma_{\text {inel }} \propto A^{2 / 3}$.

From March to November 2010 LHC collided protons at a centre-of-mass energy of $7 \mathrm{TeV}$, more than 3 times larger than the one achieved by the Tevatron $p \bar{p}$ collider. The instantaneous luminosity continuously increased over this period, passing from $L \simeq 10^{27} \mathrm{~cm}^{-2} \mathrm{~s}^{-1}$ at the beginning of March to the peak value $L=2.1 \times 10^{32} \mathrm{~cm}^{-2} \mathrm{~s}^{-1}$ in October. Such an improvement was accomplished both by increasing the number of circulating bunches - and thus reducing the time between bunch crossings - and increasing the number of protons stacked in each bunch as well as squeezing them into a smaller and smaller area. The average number of additional proton-proton (pp) interactions taking place at each bunch crossing, also known as pile-up, was negligible at the beginning and reached a maximum value of about four at the end of the data-taking period, against an expected value of 25 at full luminosity and $\sqrt{s}=14 \mathrm{TeV}$.

The last month of operations was dedicated to lead-ion collisions. The centre-of-mass energy reached the value of $2.76 \mathrm{TeV}$ per nucleon pair, more than one order of magnitude larger than the one provided by RHIC, and the instantaneous luminosity reached the value $L=3 \times 10^{25} \mathrm{~cm}^{-2} \mathrm{~s}^{-1}$.

The total integrated luminosity delivered by LHC in 2010 was about $48 \mathrm{pb}^{-1}$ with proton beams and $9.7 \mu \mathrm{b}^{-1}$ with $\mathrm{Pb}-$ ion beams.

\section{The ATLAS Detector}

ATLAS is a general-purpose detector primarily designed to study pp collisions at LHC energies. It is characterized by cylindrical symmetry, high granularity in the readout cells and coverage close to $4 \pi$, which makes it fit to study aspects of heavy-ion collisions as well. Various subsystems are arranged in layers around the beam axis, as shown in figure 1. Starting from the centre and moving outwards, ATLAS includes an Inner Detector dedicated to the reconstruction of charged- 


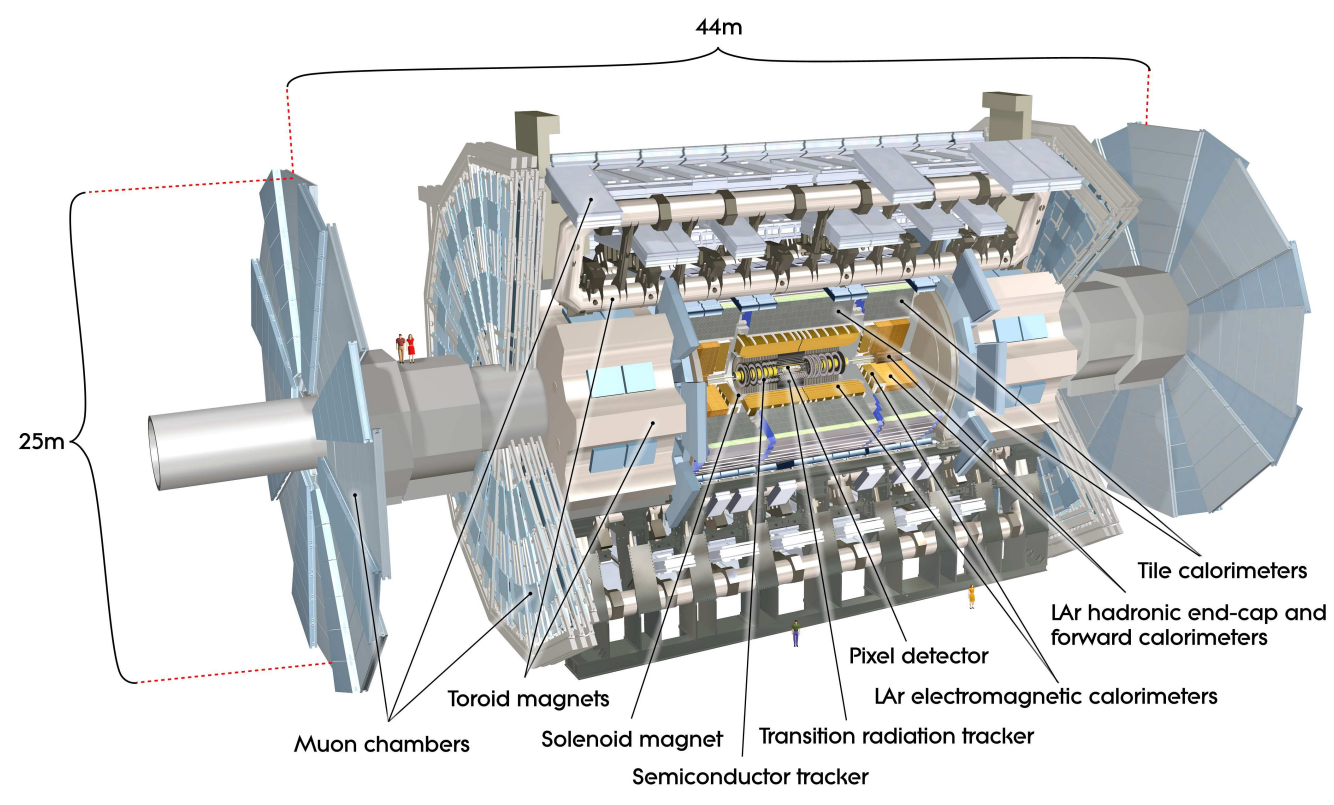

Figure 1: Cut-away view of the ATLAS detector.

particle trajectories and vertices, Calorimeters to absorb and measure the energy of photons, electrons, isolated hadrons and hadron jets, and a Muon Spectrometer to detect muons and measure their momentum. Two forward detectors have been added to the original ATLAS design: LUCID and the Zero Degree Calorimeters (ZDC), located respectively at 17 and $140 \mathrm{~m}$ from the ATLAS Interaction Point (IP), one arm per side. LUCID is dedicated to the measurement of the luminosity delivered to ATLAS by LHC, the ZDC measure neutral particles emitted at zero degrees with respect to the beam axis.

Two magnetic systems are present in ATLAS to bend the trajectory of charged particles and allow the measurement of their momentum: the Inner Detector is inside a solenoidal magnet providing a $2 \mathrm{~T}$ axial field, while toroidal magnets provide $\mathrm{B}$-fields with a peak value of $4.1 \mathrm{~T}$ in the regions of the Muon Spectrometer. A detailed description of the various subsystems can be found in [1].

The overall dimensions of ATLAS are quite impressive: it is $44 \mathrm{~m}$ long and has a diameter of $25 \mathrm{~m}$. The ATLAS Collaboration is huge as well, spanning over all inhabitated continents and gathering together 174 Institutes, for a total of about three thousand physicists.

\subsection{Trigger and Data Acquisition Performance in 2010}

Reading out a detector may involve some dead time, during which further events are lost. In case of very high acquisition rates, data storage can also become a serious issue.

In 2010 the average bunch-crossing rate of LHC in pp operations was close to $1 \mathrm{MHz}$ (it will 
approach $\sim 40 \mathrm{MHz}$ at design luminosity), and an online way to identify interesting events was clearly needed. When studying pp collisions, we are mostly interested in events where hard interactions between proton constituents occur. These produce particles at high momentum transverse to the beam axis. The other, much more frequent, events on the contrary produce most of the activity close to the beam line. The ATLAS online event selection is realized in three stages. At each level, events are kept in pipelines waiting for the decision of the following step. Loose granularity information from the calorimeters and the muon spectrometer are the main input to the first trigger level, whose average rate was about $20 \mathrm{kHz}$ in 2010 . At the second level, portions of each event are studied at full granularity. In 2010, the rate of events accepted by the second trigger level was decreased to about $3.5 \mathrm{kHz}$. Finally, the third trigger level is based on full analysis of complete events. The typical rate of pp collision events written to disk in 2010 was about $300 \mathrm{~Hz}$, higher than the design rate of $200 \mathrm{~Hz}$.

Due to lower luminosity, the trigger configuration for $\mathrm{Pb}$-ion running was aimed at avoiding biasing the selected sample. The main input to the first trigger level came from ZDC. The other trigger levels were used to reject background like beam-gas interactions, but no selection based on physics objects was applied.

The overall status of ATLAS data-taking during the whole 2010 running period was excellent: more than $97 \%$ of the millions of readout channels present in ATLAS were operational, and the overall data taking efficiency was close to $95 \%$ in both proton and lead-ion running. The fraction of good-quality data was also close to $100 \%$ for most of the subsystems.

\subsection{Inner Detector Performance}

The Inner Detector consists of three subsystems: a pixel detector, a silicon strip tracker (SCT) and a transition radiation straw-tube tracker (TRT) that also distinguishes between electrons and hadrons by measuring the amplitude of signals due to both ionization and transition radiation. The tracking efficiency as a function of transverse momentum $\left(p_{\mathrm{T}}\right)$, averaged over all pseudorapidity, rises from $\simeq 10 \%$ at $100 \mathrm{MeV}$ to $\simeq 86 \%$ for $p_{\mathrm{T}}$ above a few $\mathrm{GeV}$. The resolution on the position of the primary vertex enables the identification of multiple interactions in the same bunch crossing on an event-by-event basis, as made clear in figure 2. Secondary vertices such as those produced by B-hadrons, that typically travel a few mm before decaying, can be resolved as well. Samples of events enriched in B-hadrons can also be obtained by selecting events with tracks having high impact parameter with respect to the primary vertex, as shown in figure 3.

The nominal transverse momentum resolution:

$$
\sigma_{p_{\mathrm{T}}} / p_{\mathrm{T}} \simeq 3.8 \times 10^{-4} p_{\mathrm{T}}(\mathrm{GeV}) \oplus 0.015
$$

was studied on early pp data by reconstructing well-known particles like $K_{\mathrm{S}}^{0}, \phi, K^{*}, \Xi, \Lambda$ barions and $\mathrm{D}$ mesons. The momentum scale is known at the per mil level in the corresponding momentum range, as verified from the resolution in the measured invariant mass of the aforementioned particles. Photon conversions are particularly suitable to track the distribution of dead material and 


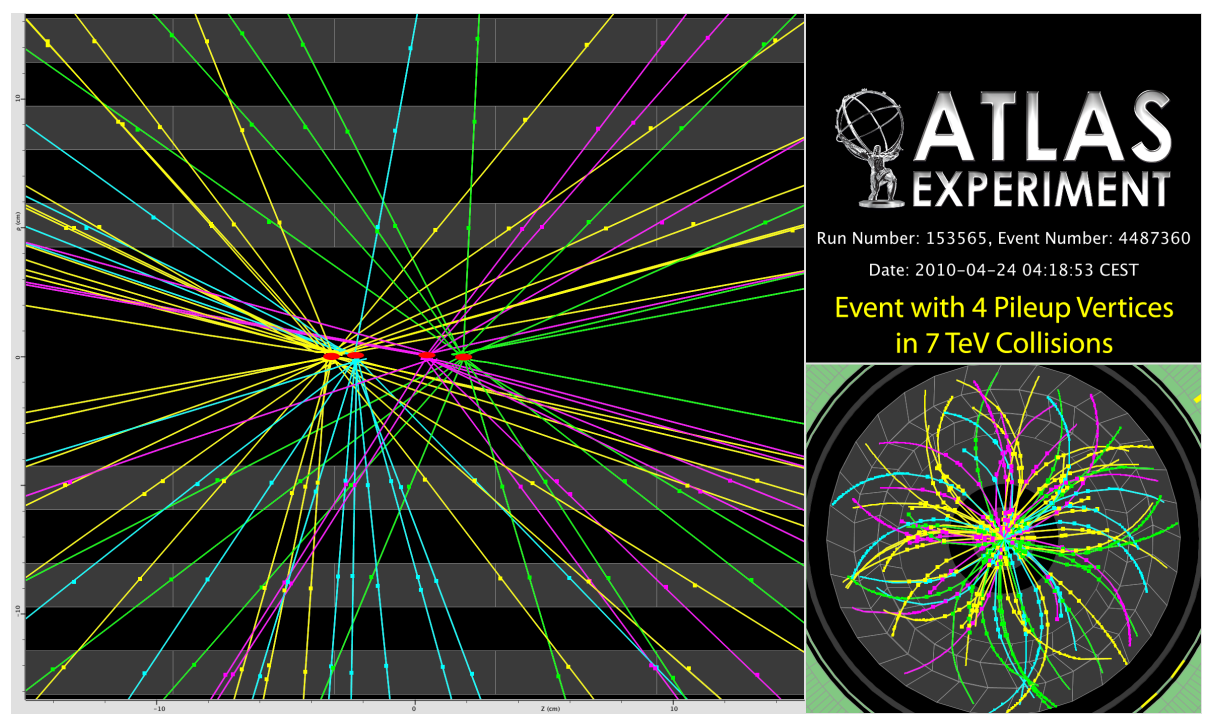

Figure 2: A pp collision event at $7 \mathrm{TeV}$ recorded by ATLAS where four different interactions are piling up. The zoomed image shows the reconstructed primary vertices.

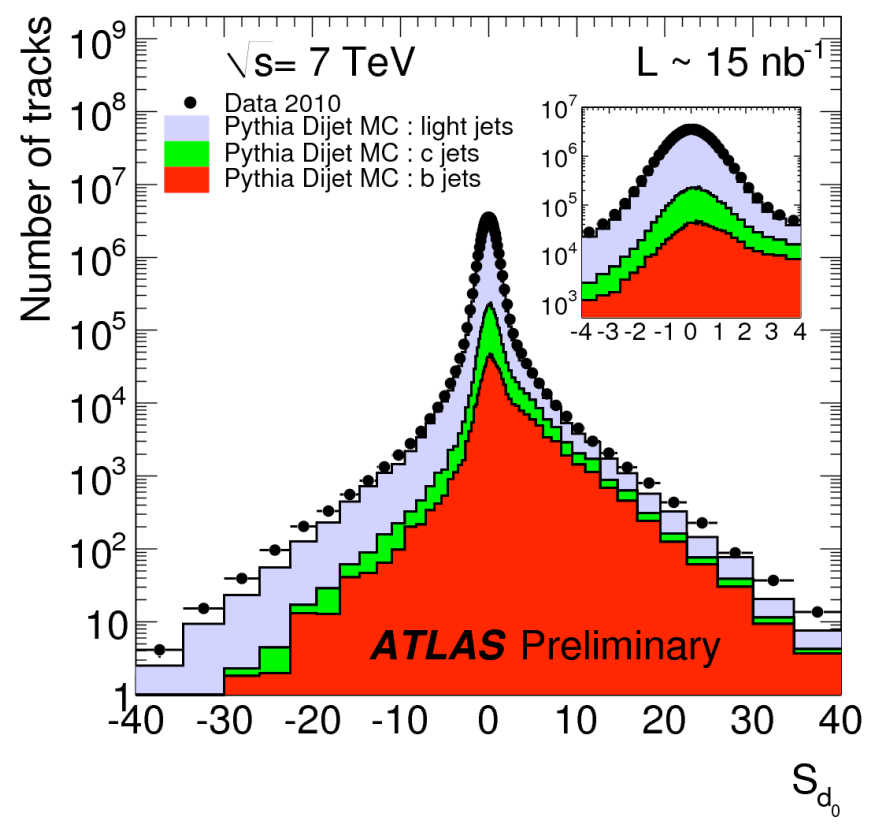

Figure 3: Distribution of the signed transverse impact parameter significance $S_{d_{0}}$ for tracks originating from different sources. Points represent data, the colored histograms Monte Carlo predictions. 
have been used to tune the Inner Detector simulation program.

Events produced in $\mathrm{Pb}$-ion collisions are characterised by particle multiplicities much larger than the ones found in pp events. As a consequence, the performance of the Inner Detector is not as good as in pp collisions, and different tracking algorithms are used. Nevertheless, the agreement between data and simulated events with respect to the average number of hits found in the various subsystems of the Inner Detector is rather good both for pp and $\mathrm{Pb}$-ion collision events, meaning that we have a good understanding of the detector behaviour in both conditions.

\subsection{Calorimeter Performance}

The ATLAS Calorimeters are located just outside the Inner detector. They must absorb and measure the energy of photons, electrons, isolated hadrons and hadron jets. They also allow the measurement of the missing transverse energy carried by high momentum neutrinos or, possibly, new weakly interacting particles.

The Electromagnetic Calorimeter uses lead as absorber and liquid argon as the active medium, which is robust against radiation doses. An accordion geometry was chosen to minimize dead areas. By neglecting the noise term, the ultimate energy resolution can be written as:

$$
\sigma_{E} / E \simeq 10 \% / \sqrt{E} \oplus 0.7 \%
$$

The Hadron Calorimeter is made of iron and scintillating tiles in the central region, where radiation doses are moderate. More radiation-hard technologies are used in the forward region, where liquid-argon is again used as the sensitive material. The ultimate energy resolution can be described as:

$$
\sigma_{E} / E \simeq 50 \% / \sqrt{E} \oplus 3 \%
$$

Electron and photon identification is based on the presence of energy clusters in the electromagnetic calorimeter, and little or no energy in the hadron calorimeter. A track in the inner detector pointing to the electromagnetic cluster is expected for electrons, whereas no tracks are present for photons. As an example of the photon identification capability of ATLAS, the top part of figure 4 shows the measured invariant mass of diphoton candidates selected in pp collision events in the region around the $\pi^{0}$ mass. Good electron identification performance allowed us to reconstruct all known $e^{+} e^{-}$resonances from a few $\mathrm{GeV}(\mathrm{J} / \psi)$ up to the $Z$ boson. The distribution of the measured invariant mass of $e^{+} e^{-}$candidates in the region around the $\mathrm{Z}$ boson mass is shown at the bottom of figure 4 .

Electrons from $\mathrm{Z}$ boson decays have $p_{\mathrm{T}}$ in the tens of $\mathrm{GeV}$ range and can be easily identified even in the high occupancy environment of $\mathrm{Pb}$-ion collisions. As an example, figure 5 shows a $\mathrm{Z}$ boson candidate produced in a $\mathrm{Pb}-$ ion collision at $\sqrt{s}_{\mathrm{NN}}=2.76 \mathrm{TeV}$. Massive gauge boson production had never been observed at other heavy-ion colliders.

Diphoton and dielectron invariant mass spectra covering the range from tens of $\mathrm{MeV}$ to hundreds of $\mathrm{GeV}$ are exceptionally sensitive calibration data. They were used to understand the align- 

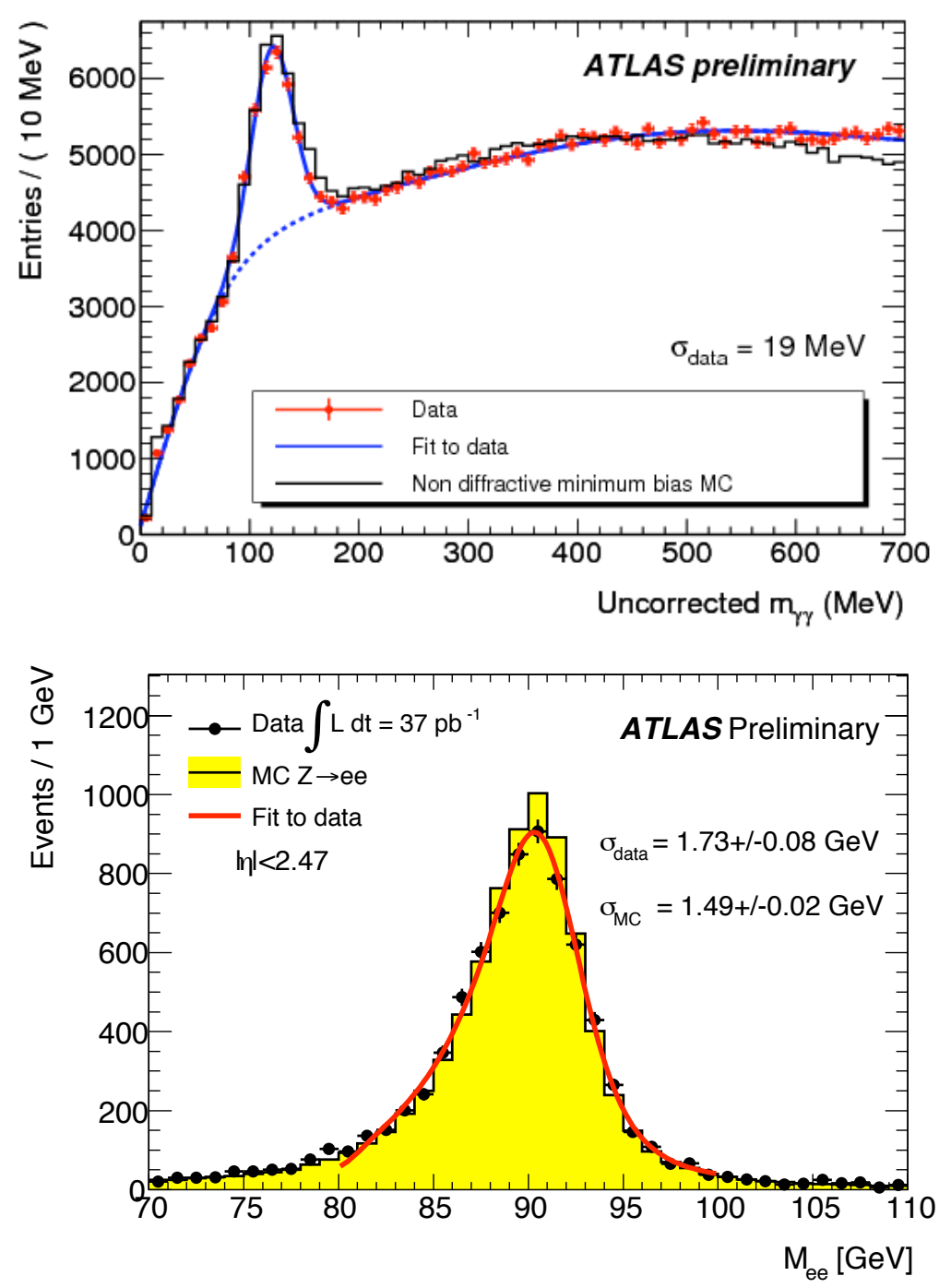

Figure 4: Top: Diphoton invariant mass spectrum in the region around the $\pi^{0}$ mass. A fit is superimposed to the points representing data. The histogram refers to Monte Carlo prediction, where the number of entries is normalized to the one found in data. Tighter photon selection cuts would be needed to make the $\eta$ peak visible. Bottom: Invariant mass distribution of $e^{+} e^{-}$candidates in the region of the $Z$ mass. Black points represent data, the histogram Monte Carlo prediction.

ment and energy resolution of the Electromagnetic Calorimeter.

Hadron jets are reconstructed with the anti- $k_{t}$ algorithm [3] starting from clusters of cells of energy above a set threshold in the hadron calorimeter. The energy resolution depends on both the jet transverse momentum and the jet pseudorapitidy. The overall precision on the energy scale is currently $\simeq 7 \%[4]$. It is expected to reach the 3-4\% level once ongoing studies with beam data on jet-balance and $E / p$ measurements for isolated hadrons will have been finalized. The impact of pile-up will also be quantitatively evaluated. To give an idea of the $p_{\mathrm{T}}$ range of jets produced at 


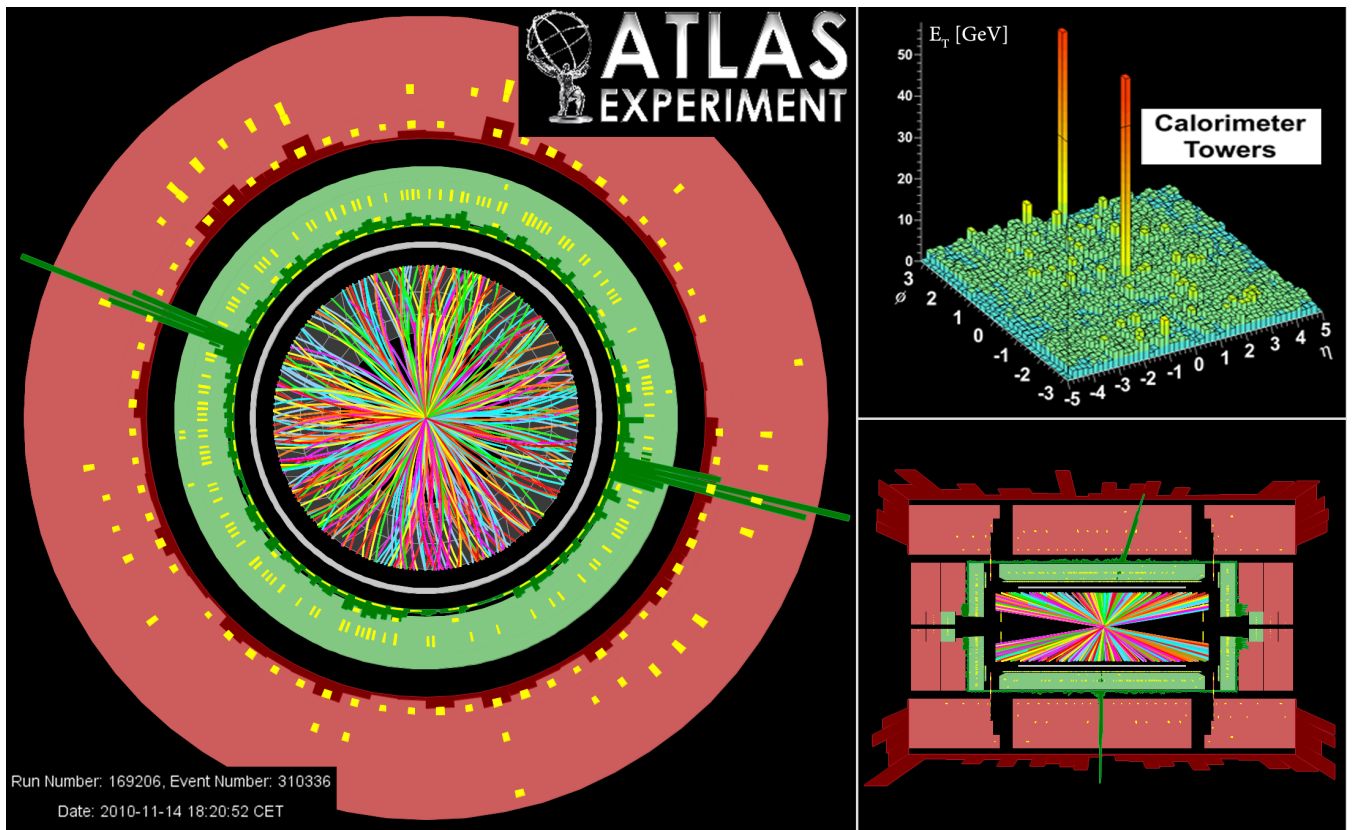

Figure 5: $\mathrm{Pb}$-ion collision event containing a $Z \rightarrow e^{+} e^{-}$candidate. The two calorimeter towers represent large and localized energy depositions in the EM calorimeter, with an invariant mass of $\simeq 104 \mathrm{GeV}$. A large number of tracks is found in the Inner Detector

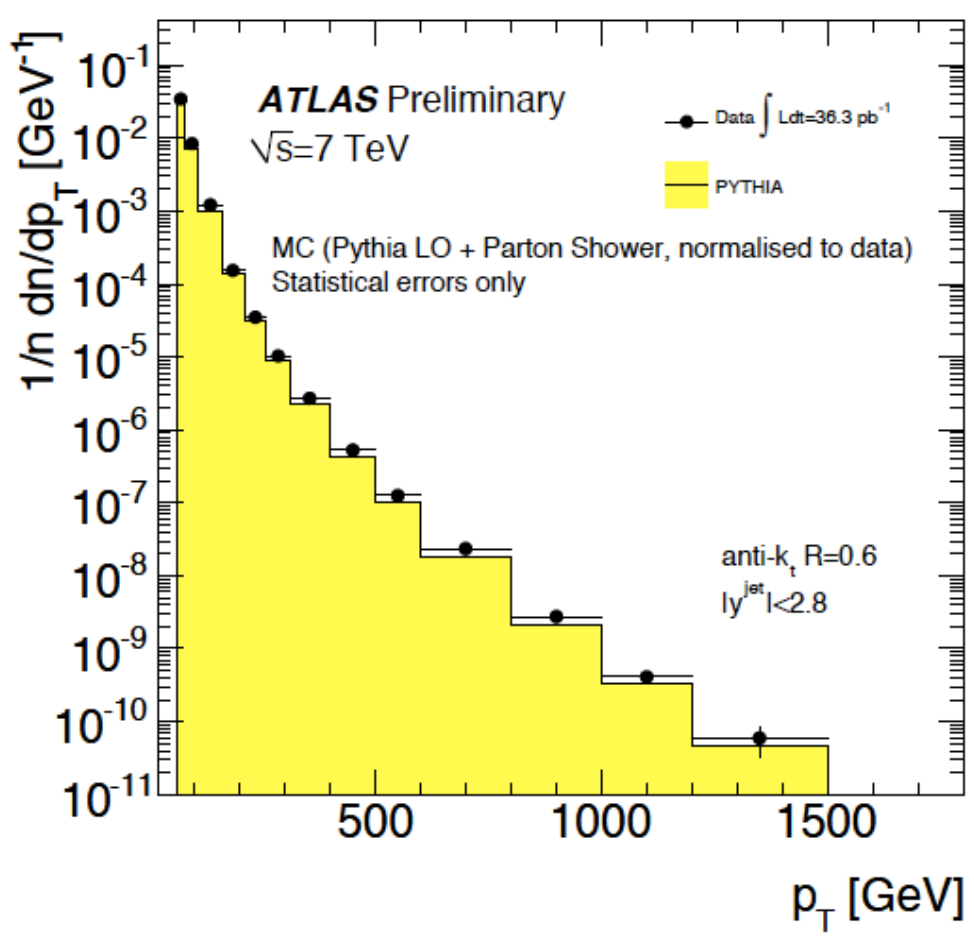

Figure 6: Distribution of the transverse momentum of jets as reconstructed in the data (black points) and as expected from Monte Carlo simulation (histogram). 


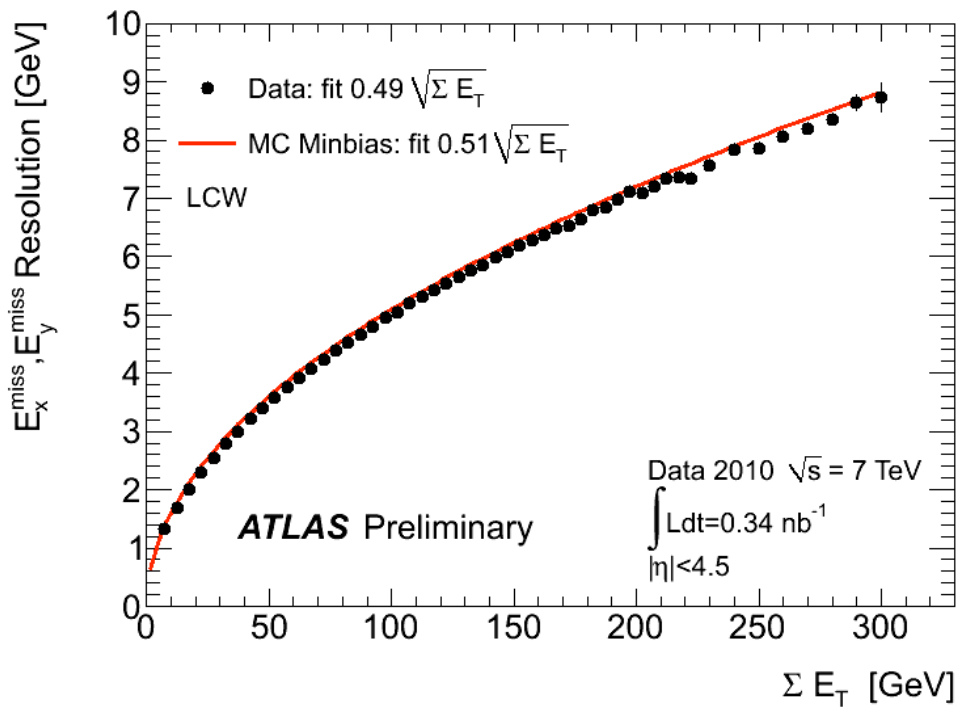

Figure 7: Resolution of the transverse missing energy $\left(E_{x}^{\text {miss }}, E_{y}^{\text {miss }}\right)$ as a function of the total transverse energy $\left(\Sigma E_{\mathrm{T}}\right)$ for pp data taken at $7 \mathrm{TeV}$. Full dots represent data while the line is a fit to the resolution obtained in Monte Carlo simulations.

LHC, figure 6 shows its distribution as measured with the data and as expected from Monte Carlo simulation. A remarkable agreement over nine orders of magnitude is apparent.

The calorimers also allow the measurement of the missing transverse energy $\left(E_{\mathrm{T}}^{\mathrm{miss}}\right)$. Its good resolution as a function of the total visible energy is shown in figure 7.

\subsection{Muon Spectrometer Performance}

The muon spectrometer consists of air-core toroidal magnets to bend muon trajectories over large areas, and drift chambers to sample their position. A minimum momentum of $\simeq 3 \mathrm{GeV}$ is required for muons to emerge from the upstream material. The detector has the capability of measuring the momentum of muons without the help of the Inner Detector, with nominal resolution $\sigma_{p_{\mathrm{T}}} / p_{\mathrm{T}}<3 \%$ up to $200 \mathrm{GeV}$ and $\sigma_{p_{\mathrm{T}}} / p_{\mathrm{T}} \simeq 10 \%$ at $1 \mathrm{TeV}$. Matching to the inner detector can obviously improve the momentum resolution and reduce the background from kaon decays as well as non-containment of hadrons within the calorimeters. As a demonstration of the good performance of the spectrometer, figure 8 shows the invariant mass of $\mu^{+} \mu^{-}$pairs selected in $\mathrm{pp}$ events. All known resonances, from the low energy ones up to the $Z$, are clearly visible. Muons can be cleanly selected in $\mathrm{Pb}$-ion collisions as well: the invariant mass of $\mu^{+} \mu^{-}$pairs in the mass region around the $Z$ mass is shown in figure 9 .

\subsection{Forward Detectors in 2010}

LUCID, a Cherenkov detector specifically designed for measuring the luminosity in ATLAS, has been operational since the first beam. It is made up of two arms, each surrounding the beampipe at $17 \mathrm{~m}$ from the ATLAS IP. Each arm contains sixteen optically reflecting aluminum tubes filled 


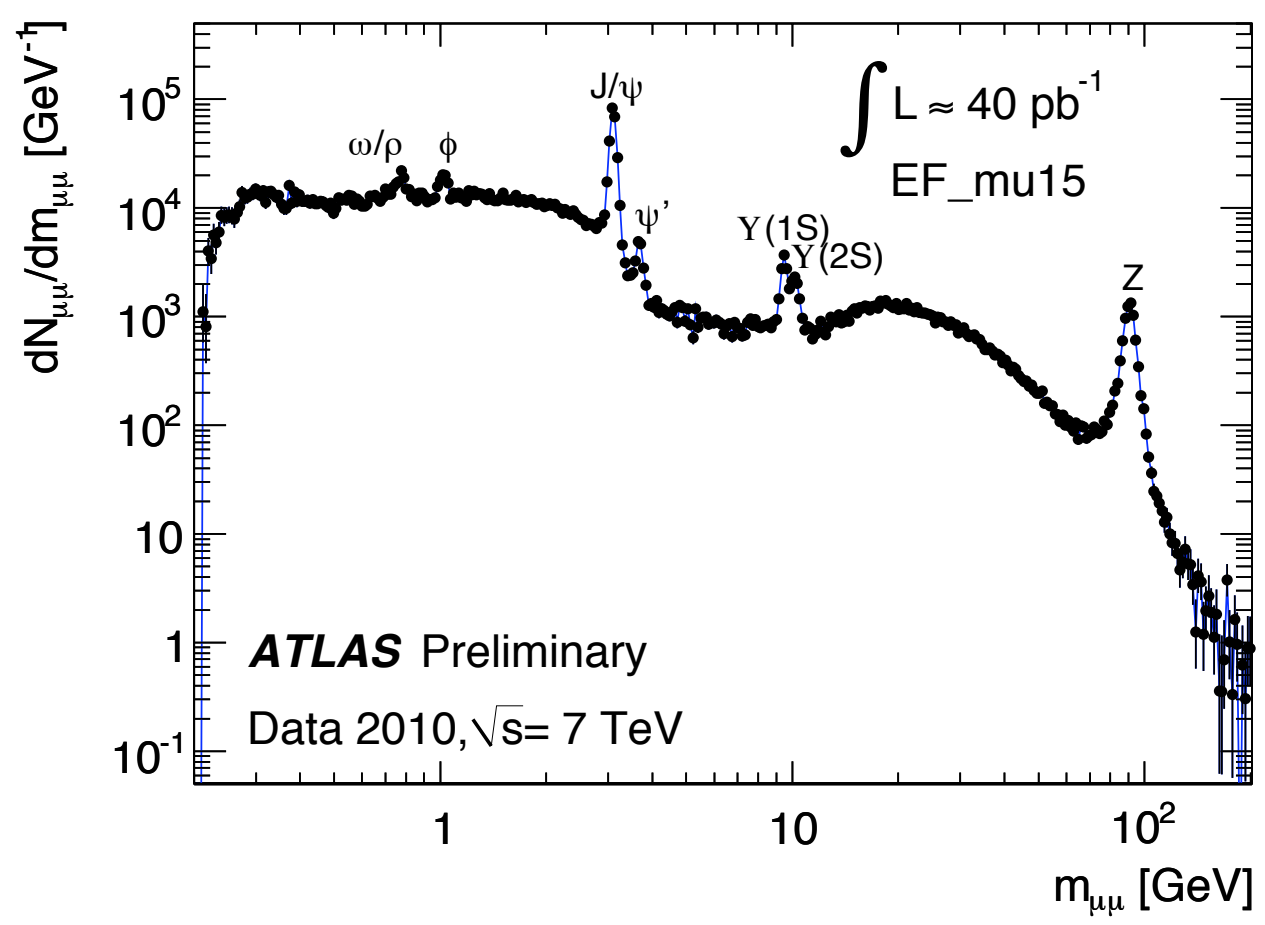

Figure 8: Invariant mass distribution of opposite-sign muon candidates selected in pp collision data at $7 \mathrm{TeV}$.

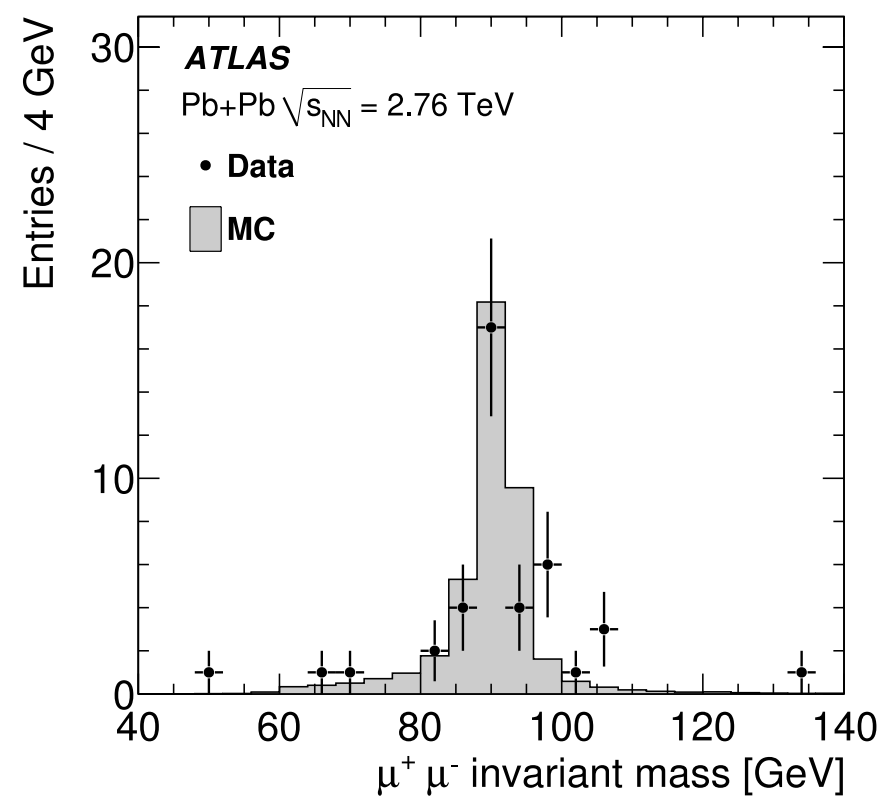

Figure 9: Invariant mass distribution of opposite-sign muon candidates as selected in $\mathrm{Pb}$-ion collision data at $\sqrt{s}_{\mathrm{NN}}=2.76 \mathrm{TeV}$. 


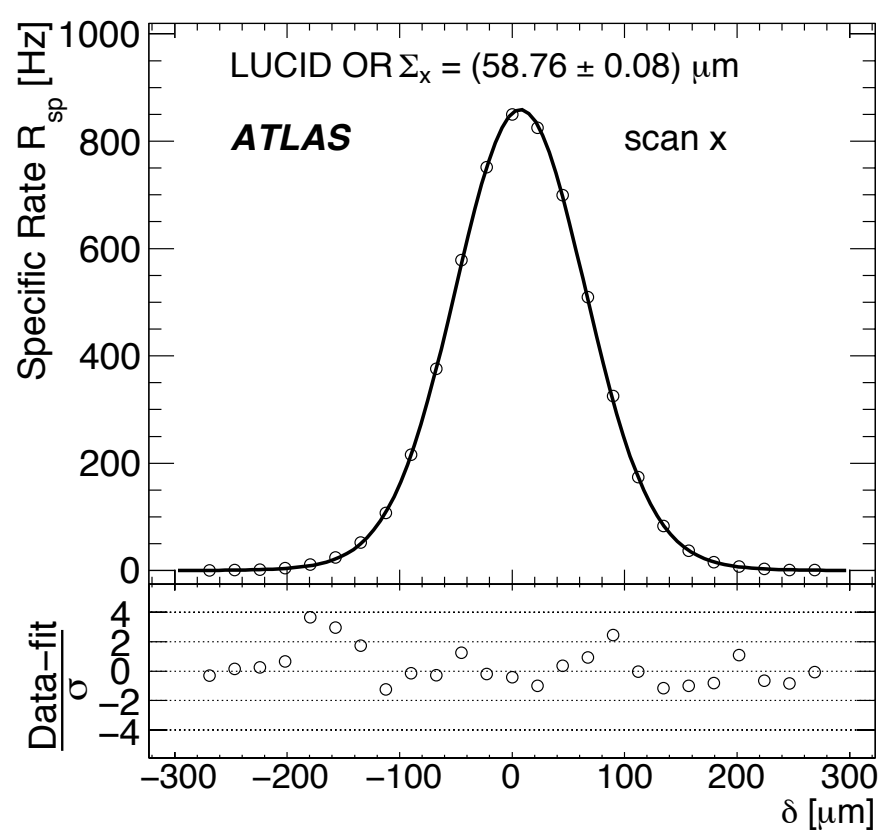

Figure 10: Specific interaction rate, defined as the measured rate divided by the product of the beam currents, versus nominal beam separation for one of the luminosity algorithms implemented by LUCID during scan III. The residual deviation of the data from the fit assuming statistical errors only is shown in the bottom panel.

with $\mathrm{C}_{4} \mathrm{~F}_{10}$. Cherenkov photons created by charged particles in the gas are reflected by the tube walls and detected by photomultipliers. The electronics of LUCID implement various algorithms to monitor the luminosity delivered by LHC (before any trigger decision) in individual bunches. All algorithms need an independent calibration to provide absolute values of delivered luminosity.

The Zero Degree Calorimeters (ZDC) were only partially installed in 2010. The detector commissioning continued throughout the proton run. The primary purpose of ZDC is to detect forward neutrons and photons with pseudorapidity $|\eta|>8.3$ in both pp and $\mathrm{Pb}$-ion collisions. The ZDC consists of two arms located at $140 \mathrm{~m}$ from the IP in slots in the LHC TAN (Target Absorber Neutral) [2], occupying space that would otherwise contain inert copper shielding bars.

\section{Measurement of Luminosity}

One of the first measurements to be performed at any accelerator is the one of luminosity relating the number of any kind of events to their production cross section.

The proton-proton total cross section is not well known in the new energy range covered by LHC and cannot be used to extract the luminosity from the total event rate with sufficient precision. However, the instantaneous absolute luminosity $L$ can also be obtained from the knowledge of 
bunch currents and beam transverse sizes:

$$
L=\frac{n_{\mathrm{b}} f_{\mathrm{r}} I_{1} I_{2}}{2 \pi \Sigma_{x} \Sigma_{y}}
$$

where $I_{1,2}$ represent individual bunch currents in the two beams, assumed to be the same for all bunches in the same beam, $n_{\mathrm{b}}$ is the number of circulating bunches, $f_{\mathrm{r}}$ the bunch crossing frequency and $\Sigma_{x(y)}$ the transverse dimension of head-on beams. In 2010, the absolute luminosity was mesured with the technique of van der Meer [5] in dedicated scans, and used to calibrate all ATLAS subsystems capable of acting as luminosity monitors. During a van der Meer scan the beams are moved with respect to each other in the directions perpendicular to the beam line, while luminosity monitors count the number of interactions produced. By fitting the bell-shaped curve representing the measured rate as a function of the beam displacement, as shown for example in figure 10, the beam transverse size $\Sigma_{x(y)}$ can be extracted from the relation:

$$
\Sigma_{x}=\frac{1}{\sqrt{2 \pi}} \frac{\int R_{x}(x) d x}{R_{x}(0)}
$$

where $R_{x}(x)$ is the measured rate at beam separation $x$ and $\int R_{x}(x) d x$ is nothing but the curve area. The absolute luminosity is then obtained by combining the beam sizes with a measurement of the beam currents.

At the time of this conference only data from three out of four scans had been analysed, providing a value for the luminosity that is fully dominated by the uncertainty on the beam currents as provided by LHC [6]. Once the latest scan data will have been analysed, the precision on $L$ is expected to improve from the current $11 \%$ to better than $5 \%$.

A van der Meer scan was also performed during the lead-ion run. Data are still under analysis. Meanwhile the luminosity is obtained from the expected cross section, the ZDC observed rate of events and the ZDC efficiency.

\section{Physics Results from Proton-Proton Collisions}

With all the ingredients described so far, we can start to talk about physics. The selection of results presented in the following sections are to a large extent focussed on data collected in the first running period of LHC, when the pile-up probability was negligible. The delay between data collection and availability of results is due to the need to understand the detector and the effects of pile-up, as well as tune Monte Carlo simulations using real data. However, updates based on full statistics are expected soon.

The total cross section for pp collisions at $7 \mathrm{TeV}$ amounts to $\simeq 75 \mathrm{mb}$. Most of the interactions result in events characterized by the production of particles with transverse momentum not exceeding a few $\mathrm{GeV}$. Hard jets, with transverse momentum of tens of $\mathrm{GeV}$ or more, are produced in a small fraction of the collisions $\left(\simeq 10^{-5} \%\right)$. Vector boson production is equally rare, and even rarer is the production of top quarks. Given the luminosity delivered by LHC in 2010, the physics 


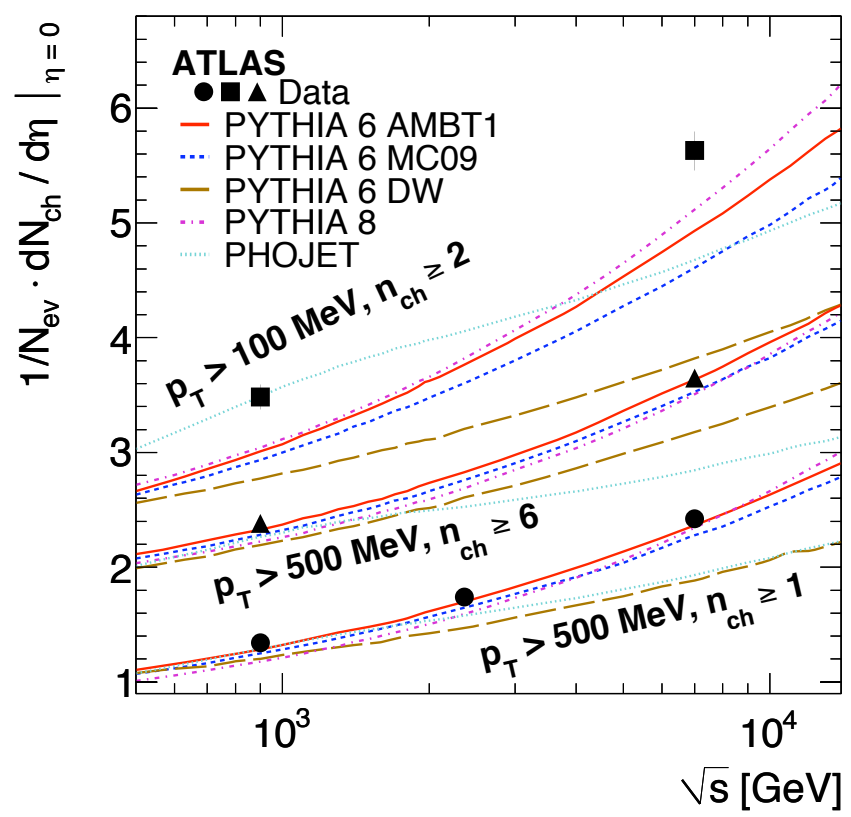

Figure 11: Average charged-particle multiplicity per unit of rapidity for $\eta=0$ as a function of the centreof-mass energy. The results with $n_{\mathrm{ch}} \geq 2$ within the kinematic range $p_{\mathrm{T}}>100 \mathrm{MeV}$ and $|\eta|<2.5$ are shown alongside the results with $n_{\mathrm{ch}} \geq 1$ within the kinematic range $p_{\mathrm{T}}>500 \mathrm{MeV}$ and $|\eta|<2.5$ at 0.9 , 2.36 and $7 \mathrm{TeV}$. The data are compared to various particle level MC predictions based on both PYTHIA [7] and PHOJET [8] generators. The vertical error bars on the data represent the total uncertainty.

studied so far is often referred to as "rediscovery of the Standard Model", although searches for new physics are starting to improve limits on production of exotic particles. The full rediscovery of the Standard Model in just one experiment was at any rate quite an exciting exercise.

\subsection{Properties of Minimum Bias Events}

The first things to study and model when entering new energy ranges are basic properties of minimum bias events, like the dependence of charged particle multiplicity on momentum, centreof-mass energy and pseudorapidity. Furthermore, since minimum bias events are overlaid on the hard ones in case of pile-up, a good modelling and comprehension of the former is mandatory to understand the latter. No first principle exists to describe quark and gluon hadronization, and model predictions must be validated by data.

ATLAS has measured the charged-particle multiplicity in pp interactions at $7 \mathrm{TeV}$, its dependence on transverse momentum and pseudorapidity as well as the relationship between the mean transverse momentum and the charged-particle multiplicity [9]. Measurements have been carried out in different regions of phase-space, providing both diffraction-reduced measurements and more inclusive ones. The observed distributions are converted to particle-level using modelindependent corrections. In any considered kinematic region the observed particle multiplicities are higher than predicted by Monte Carlo models, although the discrepancies are larger in phase- 

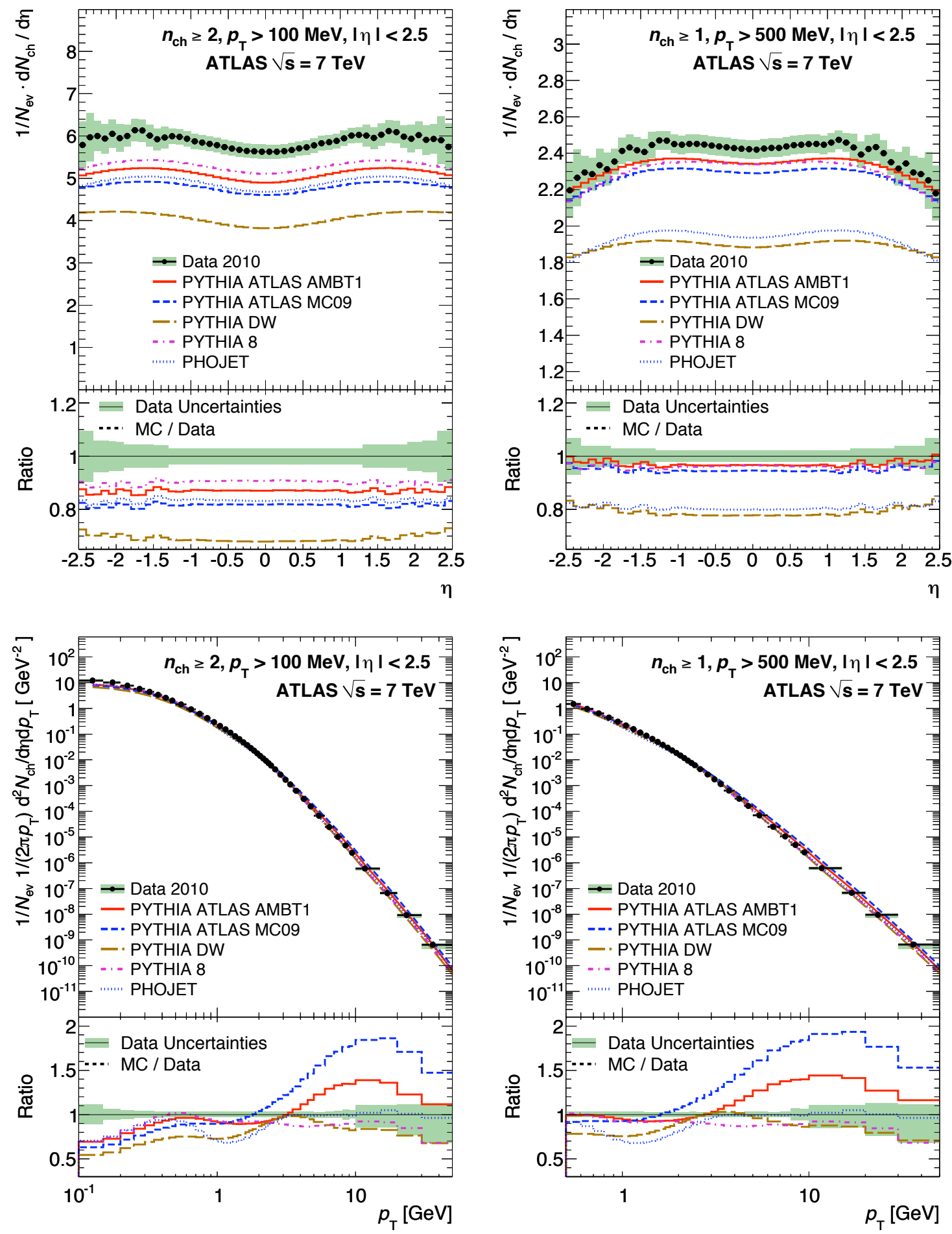

Figure 12: Top: Charged-particle multiplicities $\left(n_{\mathrm{ch}}\right)$ as a function of the pseudorapidity $(|\eta|)$ for events with tracks in various ranges of multiplicity, transverse momentum $\left(p_{\mathrm{T}}\right)$ and pseudorapidity at $\sqrt{s}=7 \mathrm{TeV}$. Bottom: Charged-particle multiplicities as a function of the transverse momentum in different phase-space regions at $7 \mathrm{TeV}$. The dots represent the data and the curves the predictions from different MC models. AMBT1 refers to a PYTHIA Monte Carlo sample tuned to ATLAS data at $900 \mathrm{GeV}$. The vertical bars represent the statistical uncertainties, while the shaded areas show statistical and systematic uncertainties added in quadrature. The bottom inserts show the ratio of the MC over the data. 

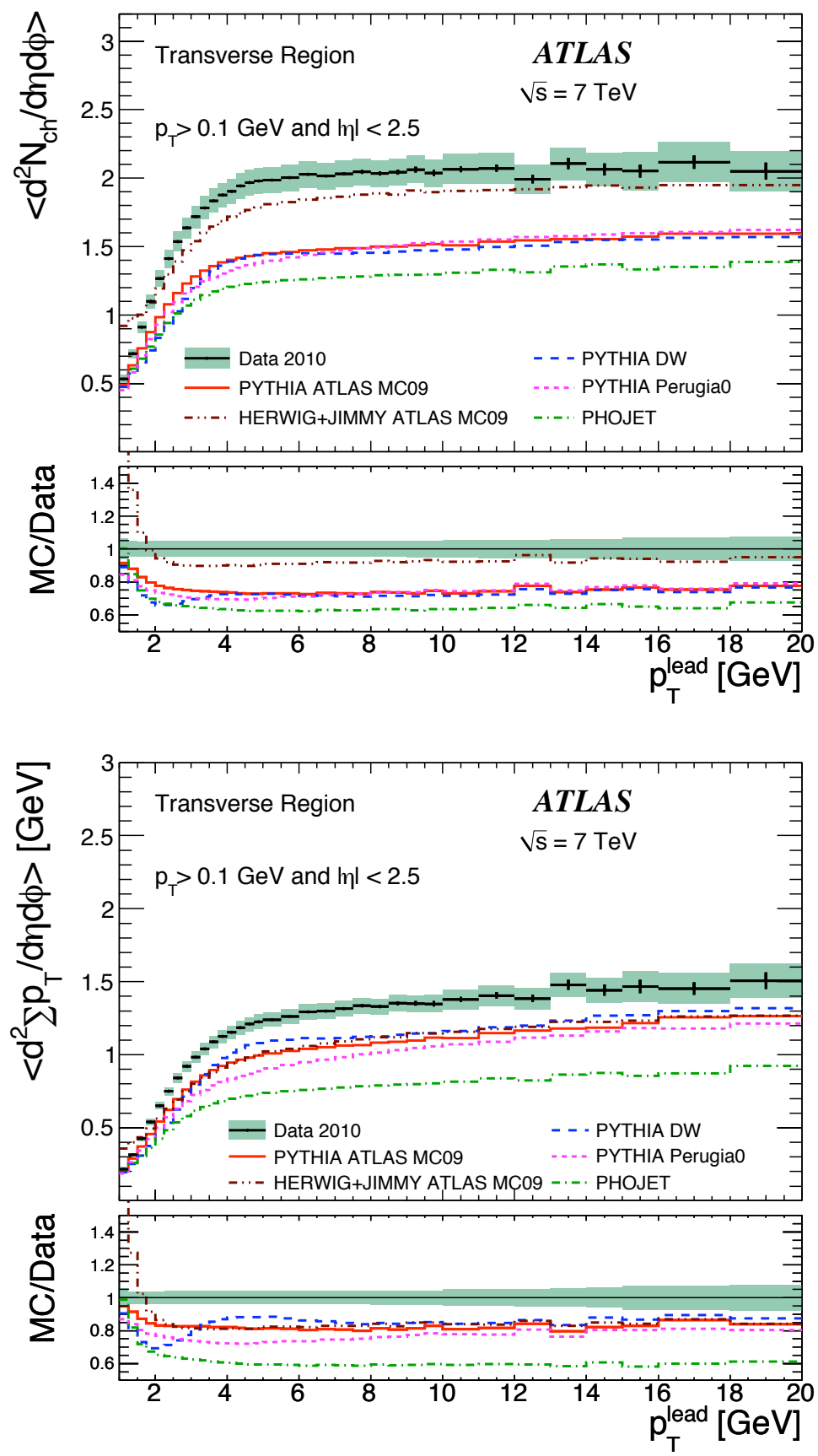

Figure 13: Density of charged particles (top) and of the scalar sum of transverse momentum of charged particles (bottom) in the underlying event as a function of the transverse momentum of the leading particle. The vertical bars represent the statistical uncertainties, while the shaded areas show the combined statistical and systematic uncertainties. 
space regions where diffraction is not suppressed, as shown in figure 11 and 12.

An alternative way to study particle hadronization is to look at the so called "underlying event". This is defined as those aspects of a hadronic interaction attributed not to the hard scattering process, but rather to the accompanying interactions of the rest of the proton. Three regions are defined in azimuthal angle with respect to the highest transverse momentum charged particle in the event (toward: $|\Delta \phi|<60^{\circ}$; transverse: $60^{\circ}<|\Delta \phi|<120^{\circ}$; away: $|\Delta \phi|>120^{\circ}$ ), such that the region transverse to the dominant momentum-flow is most sensitive to the underlying event. By studing the underlying event, interplay between hard and soft QCD can be better investigated. The latest ATLAS studies [10] point to conclusions similar to the previous ones, namely that soft-QCD models tuned to data at lower energy are unable to fully describe the same processes at the energy of LHC. As an example, figure 13 shows the density of charged particles and the density of the scalar sum of transverse momentum of charged particles in the underlying event as a function of the transverse momentum of the leading particle in the event. The ratio between model expectations and data is shown in the bottom inserts of the pictures.

\subsection{Production of Jets}

The agreement between data and theoretical predictions improves if we move to hard processes which can be described by QCD.

ATLAS has measured the inclusive single-jet differential cross section as a function of jet transverse momentum and rapidity with a data sample corresponding to an integrated luminosity of $17 \mathrm{nb}^{-1}$ at $7 \mathrm{TeV}$ [11]. Dijet cross sections have been studied as well, as a function of the dijet invariant mass and angular separation. The anti- $k_{t}$ algorithm is used to identify jets, with two jet resolution parameters, $R=0.4$ and 0.6. The results are in good agreement with expectations based on next-to-leading-order $\mathrm{QCD}$, providing a validation of the theory in the new kinematic regime covered by LHC up to now. In particular, figure 14 shows the inclusive jet differential cross section as a function of jet $p_{\mathrm{T}}$ as well as the dijet double-differential cross section as a function of dijet mass, binned in the maximum rapidity of the two leading jets. The data are compared to NLO pQCD calculations to which soft QCD corrections have been applied. The error bars indicate the statistical uncertainty on the measurement, and the grey shaded bands indicate the quadratic sum of the systematic uncertainties, dominated by the jet energy scale uncertainty. The additional uncertainty of $11 \%$ due to the luminosity measurement is not shown.

Jet shapes have been measured using $3 \mathrm{pb}^{-1}$ of data, in the transverse momentum range $30 \mathrm{GeV}<\mathrm{p}_{\mathrm{T}}<600 \mathrm{GeV}$ and rapidity $|\eta|<2.8$ [12]. The data were corrected for detector effects and compared to several leading-order QCD matrix elements plus parton shower Monte Carlo predictions, including different sets of parameters tuned to model fragmentation processes and underlying event contributions in the final state. Jets originating from gluons are expected to be broader than jets originating from quarks, due to the higher probability for gluons to radiate other gluons. Consistently, jets were observed to become narrower as their momentum increases and the contribution of gluon-initiated jets becomes smaller. For an appropriate choice of the parameters 

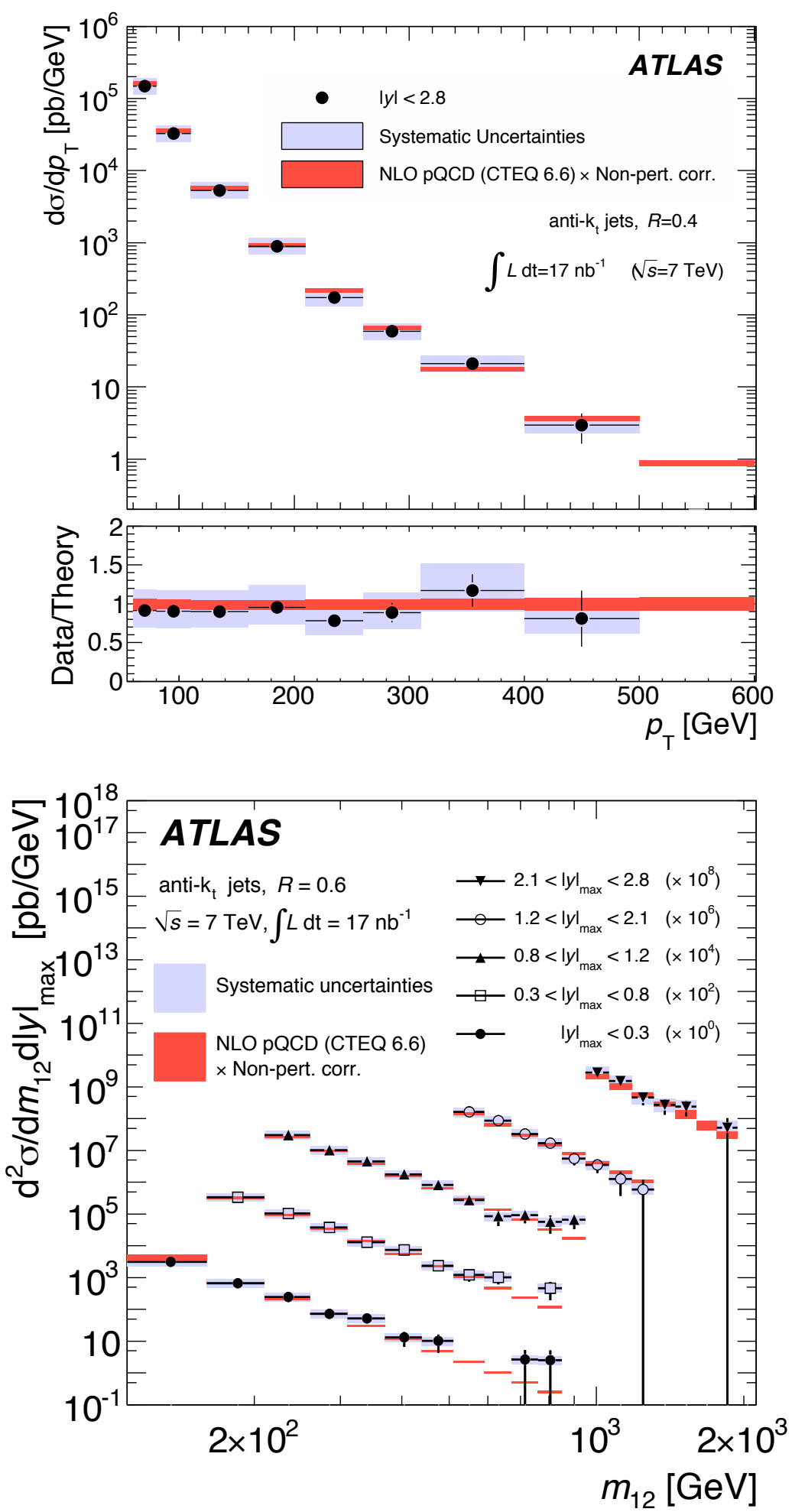

Figure 14: Top: Inclusive jet differential cross section as a function of jet $p_{\mathrm{T}}$, integrated over the pseudorapidity region $|\eta|<2.8$ for jets identified using the anti- $k_{t}$ algorithm with $\mathrm{R}=0.4$. Bottom: Dijet double-differential cross section as a function of dijet mass, binned in the maximum rapidity of the two leading jets $|y|_{\max }$, for jets identified using the anti- $k_{t}$ algorithm with $\mathrm{R}=0.6$. 

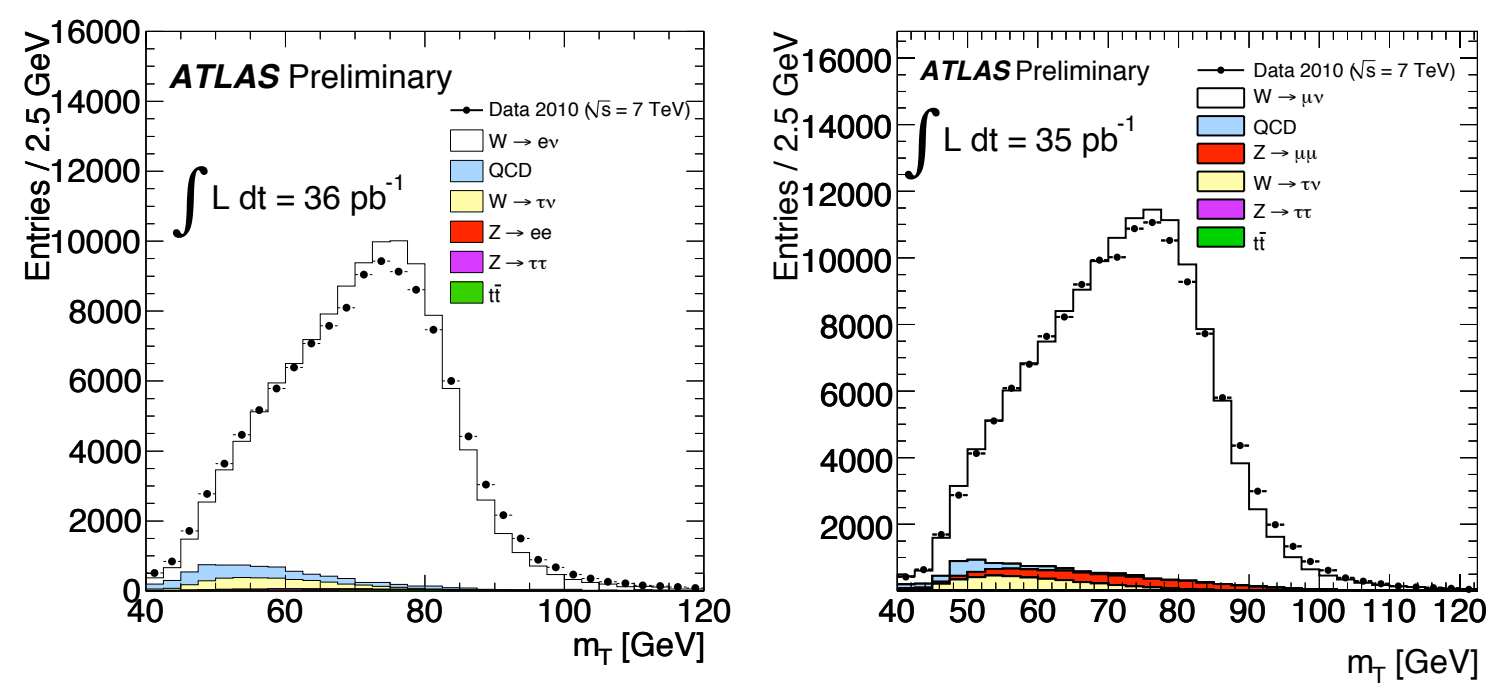

Figure 15: Distribution of the transverse mass of one electron (left) or muon (right) candidate and missingenergy as measured by ATLAS with a data sample of $35 \mathrm{pb}^{-1}$ collected in 2010 . The statistics correspond to about $11900 \mathrm{~W}$ candidates in the electron channel, and $142000 \mathrm{~W}$ candidates in the muon channel.

used in the fragmentation and underlying event models, the data appeared to be well described by Monte Carlo simulation.

\subsection{Production of Massive Gauge Vector Bosons}

$W$ and $Z$ bosons $\left(m_{W}=80.4 \mathrm{GeV}, m_{Z}=90.1 \mathrm{GeV}\right)$ are abundantly produced at LHC. The production cross section is sensitive to the proton PDF at low $x$. Both bosons are produced via quark fusion and can be cleanly detected via their leptonic decays. The neutrino from the decay of the $\mathrm{W}$ boson manifests itself as substantial missing transverse energy. As a consequence, the invariant mass of the lepton-neutrino pair coming from the $\mathrm{W}$ decay, $m_{\mathrm{T}}$, can be reconstructed only in the transverse plane:

$$
m_{\mathrm{T}}=\sqrt{2 p_{\mathrm{T}}^{\ell} E_{\mathrm{T}}^{\mathrm{miss}}(1-\cos \varphi)}
$$

where $p_{\mathrm{T}}^{\ell}$ is the transverse momentum of the lepton and $\varphi$ is the azimuthal angle between the lepton momentum and the missing energy vectors. The high edge of the distribution of the lepton-missing energy transverse mass is sensitive to the very value of the $\mathrm{W}$ mass. Figure 15 shows such distributions for the $W \rightarrow e v$ and $W \rightarrow \mu v$ channels as measured by ATLAS with a data sample of $\sim 35 \mathrm{pb}^{-1}$ collected in pp collisions in 2010 .

The study of massive vector boson $(V$, where $V=W$ or $Z$ ) production in association with one or more jets $(V+$ jets $)$ is an important test of quantum chromodynamics. In addition, $V+$ jets processes are a significant background to studies of Standard Model processes such as $t \bar{t}$ or single-top production, as well as searches for the Higgs boson and for physics beyond the Standard Model. Measurements of the cross section and kinematic properties of $V+$ jets processes and comparisons to theoretical predictions are therefore of significant interest. 

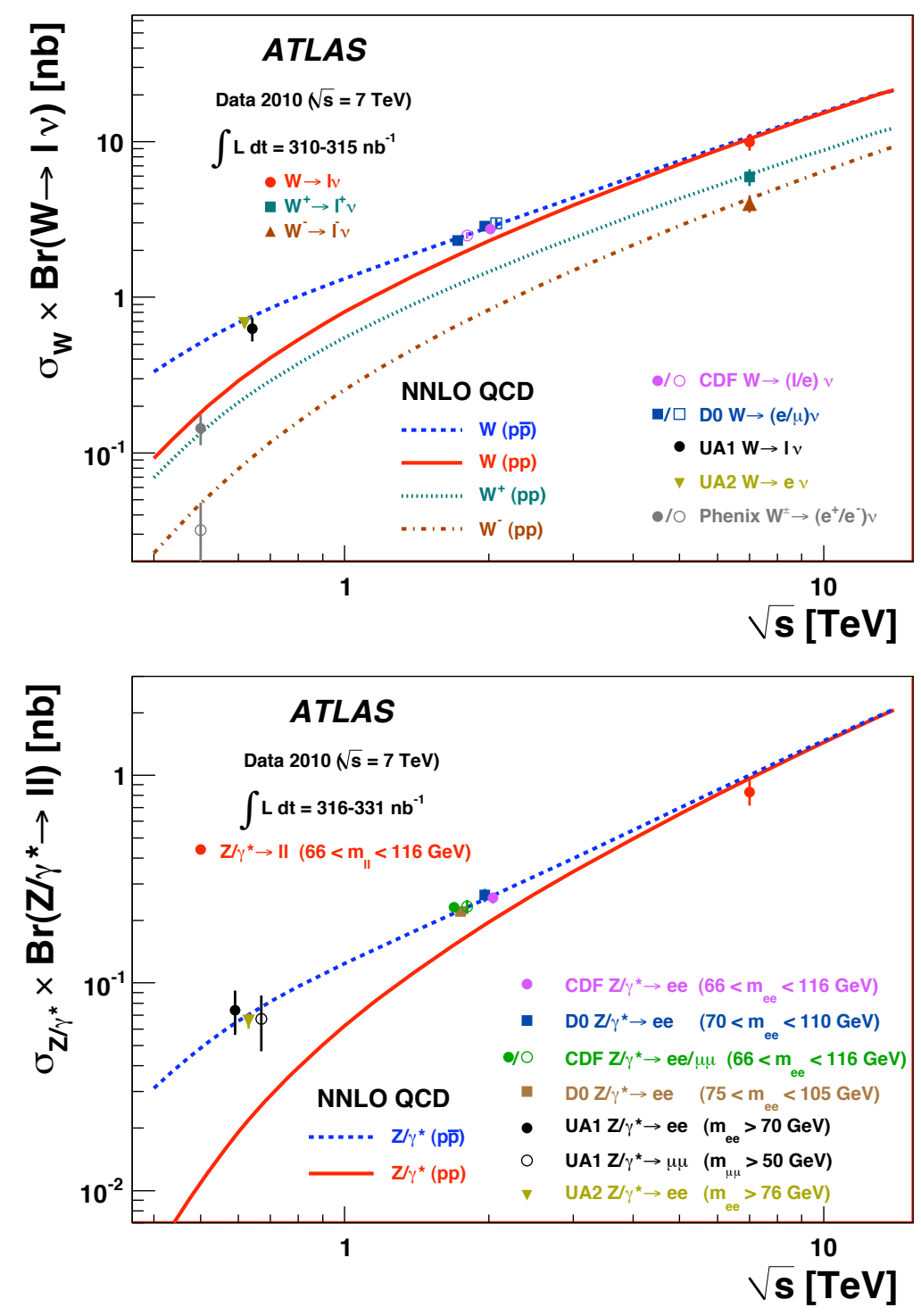

Figure 16: Top: The measured values of $\sigma_{W} \times B R(W \rightarrow \ell v)$ for $W^{+}, W^{-}$and for their sum . Results are shown for the combined electron-muon results. Bottom: The measured value of $\sigma_{Z} / \gamma^{*} \times B R\left(Z / \gamma^{*} \rightarrow \ell^{+} \ell^{-}\right)$ where the electron and muon channels have been combined. Theoretical predictions based on NNLO QCD calculations are shown for both proton-proton and proton-antiproton colliders as a function of $\sqrt{s}$. In addition, previous measurements at proton-antiproton colliders are shown. The data points at the various energies are staggered to improve readability. The CDF and D0 measurements are shown for both Tevatron collider energies, $\sqrt{s}=1.8 \mathrm{TeV}$ and $\sqrt{s}=1.96 \mathrm{TeV}$. All data points are displayed with their total uncertainty. The theoretical uncertainties are not shown. 

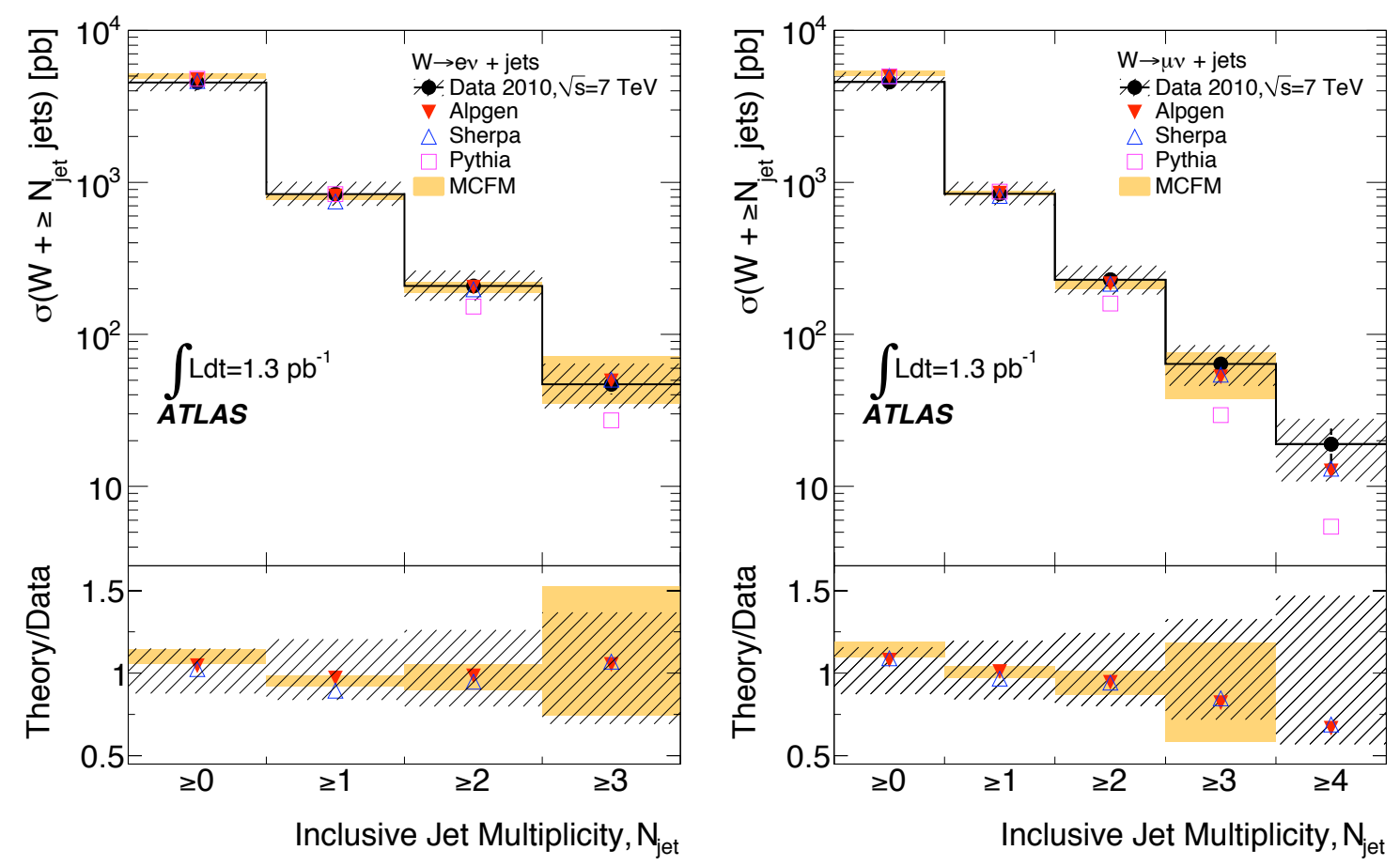

Figure 17: $\mathrm{W}+\mathrm{jets}$ cross-section as a function of jet multiplicity. The $\mathrm{W}$ boson is selected in either the $e v$ (left) or $\mu \nu$ (right) decay channel. Statistical uncertainties in the data are shown by the vertical bars. The combined statistical and systematic uncertainties are shown by the hashed regions. The uncertainties are correlated from bin to bin. Also shown are predictions from PYTHIA, ALPEN [15], SHERPA [16] and MCFM [17], and the ratio of theoretical predictions to data. The theoretical uncertainties are shown only for MCFM, which provides NLO predictions for $N_{\text {jets }} \leq 2$ and LO predictions for $N_{\text {jets }}=3$.

ATLAS has measured the $W \rightarrow \ell v$ and $Z / \gamma^{*} \rightarrow \ell^{+} \ell^{-}(\ell=e, \mu)$ production cross sections times leptonic branching ratio with a pp data set corresponding to an integrated luminosity of approximately $320 \mathrm{nb}^{-1}$ [13]. The results are based on $2250 \mathrm{~W} \rightarrow \ell v$ and $179 \mathrm{Z} / \gamma^{*} \rightarrow \ell^{+} \ell^{-}$and are shown in figure 16 together with measurements at lower energy as well as theoretical predictions. Measurements of the $W^{+}$and $W^{-}$production cross sections agree with expectations on charge asymmetry. The measurements are currently limited by the uncertainty on the luminosity.

Based on an integrated luminosity of $1.3 \mathrm{pb}^{-1}$, ATLAS also studied the production of $\mathrm{W}$ bosons in association with jets [14]. Cross sections are presented as a function of jet multiplicity in figure 17. Next-to-leading order calculations are found to be in good agreement with the data. Leading-order multiparton event generators, normalized to the NNLO total cross section, describe the data well for all measured jet multiplicities. Analysis of the full data sample is still ongoing, although preliminary studies, including $\mathrm{Z}$ boson plus jet candidates, show no evidence of disagreement with theoretical predictions.

At LHC gauge bosons can also be produced in pairs, although less frequently. A few events with both ZZ and WZ candidates decaying leptonically have been cleanly detected. 


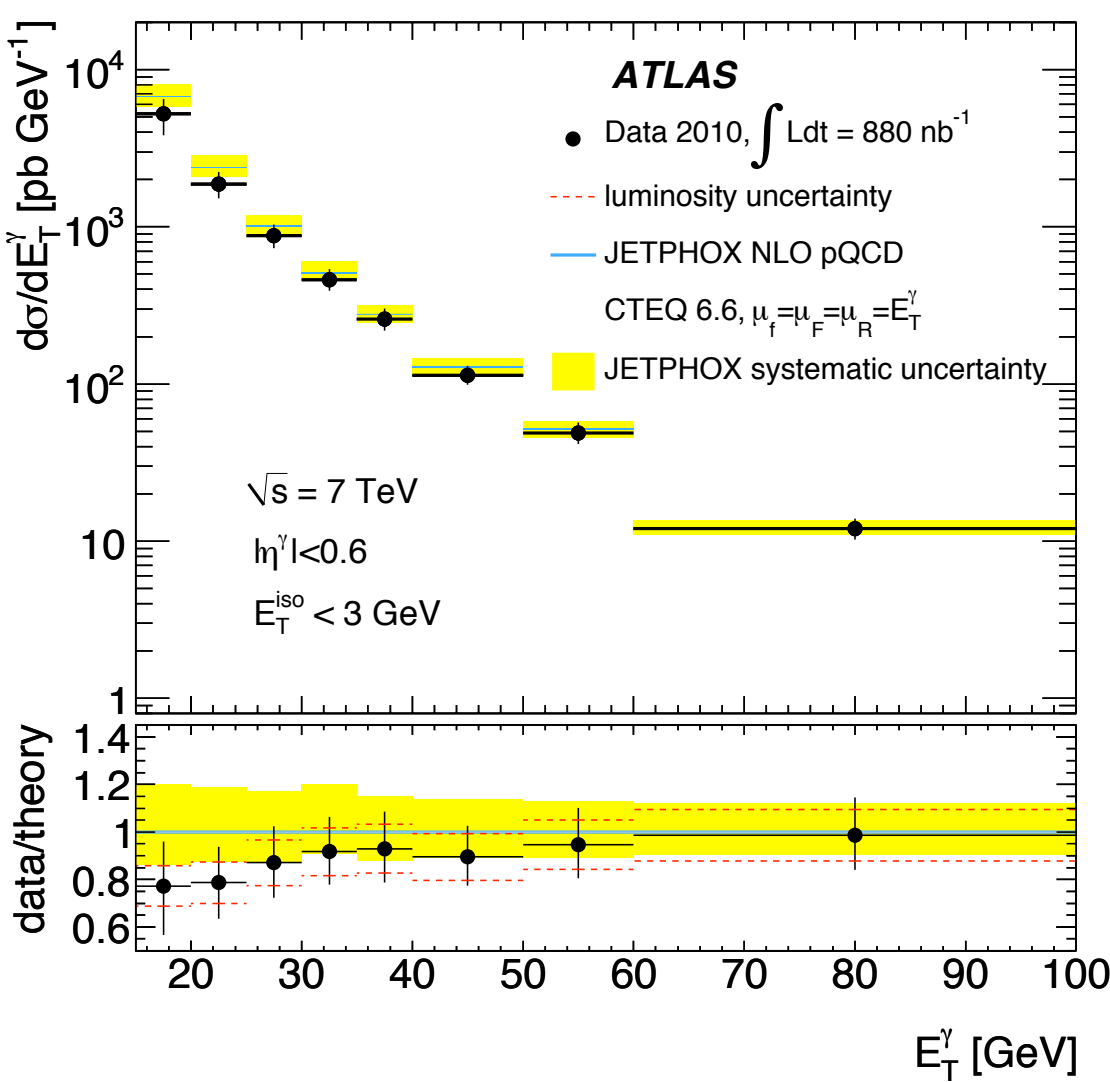

Figure 18: Measured and expected inclusive prompt photon production cross section, for photons with transverse energies above $15 \mathrm{GeV}$ and in the pseudorapidity range $|\eta|<0.6$. The band represents the total systematic uncertainty on the JetPhox NLO pQCD prediction. The error bars on the data represent the combination of all statistical and systematic uncertainties, except for the uncertainty on the luminosity measurement, which is indicated by the dashed lines.

\subsection{Isolated Prompt Photons}

Prompt photon production at hadron colliders provides a handle for testing perturbative QCD (pQCD) predictions. Photons provide a colorless probe of quarks in the hard partonic interaction and the subsequent parton shower. Their production cross section is sensitive to the gluon content of the proton, so that measuring it can constrain the gluon density function. Furthermore, photon identification is important for many physics signatures, including searches for the Higgs boson, graviton decays to photon pairs, decays of excited fermions, and decays of pairs of supersymmetric particles characterized by the production of two energetic photons and large missing transverse energy. Prompt photons include both "direct photons", which take part in the hard scattering subprocess, and "fragmentation" photons. If an isolation requirement is applied, the relative contribution to the total cross section from fragmentation photons decreases, though it remains non-negligible especially at low transverse energies. 
ATLAS has measured the production cross section of isolated prompt photons in the transverse energy range $15<E_{\mathrm{T}}<100 \mathrm{GeV}$ [18] and in different rapidity regions. The results are based on an integrated luminosity of $880 \mathrm{nb}^{-1}$. Photon candidates are identified by combining information from the calorimeters and from the inner tracker. The results for the rapidity range $|\eta|<0.6$ are shown in figure 18 and agree with predictions from next-to-leading order perturbative QCD calculations.

\subsection{Production of Top Quarks}

The top quark is by far the heaviest quark, with a measured mass of $172 \mathrm{GeV}$. At LHC top quarks are mostly produced in pairs. Their mass is so high that there is no time for them to hadronize before decaying. The branching fraction to a $W$ boson and a b quark is close to $100 \%$, so that the final $t \bar{t}$ event topology is fully determined by the decay of the $W$ bosons. Events where at least one of the $W$ bosons decays leptonically can be separated from the high multi-jet QCD background with the best signal-to-noise ratio. The capability to identify jets originating from b-quarks (b tagging) is the key to rejecting $W+$ jets events where no top quarks are produced. The semileptonic channel, where one of the $\mathrm{W}$ boson decays leptonically and the other decays into a jet pair, can be used to measure the top mass via the invariant mass of the three jets coming from the $\mathrm{b} q \bar{q}$ system.

ATLAS performed a first measurement of the production cross-section for top quark pairs $(t \bar{t})$ based on a data sample of $2.9 \mathrm{pb}^{-1}$ [19]. Events were selected in two different topologies: single lepton (electron $e$ or muon $\mu$ ) with large missing transverse energy and at least four jets, and dilepton (ee, $\mu \mu$ or $e \mu$ ) with large missing transverse energy and at least two jets. 37 candidate events were observed in the single-lepton topology and 9 events in the dilepton topology, against an estimated background of 12 and 2.5 events respectively. The kinematic properties of the selected events were found to be consistent with Standard Model $t \bar{t}$ production, and the measured production cross-section shown in the top part of figure 19 was found to be in agreement with perturbative QCD calculations. The distribution of the invariant mass of the three jets giving the largest combined $p_{\mathrm{T}}$, in semileptonic $t \bar{t}$ candidate events with at least one $\mathrm{b}$-tagged jet, is shown at the bottom of figure 19 and uses $\sim 35 \mathrm{pb}^{-1}$ of pp data collected in 2010 .

\subsection{First Searches for New Physics}

First searches for new physics included heavy particles decaying into jets, for which the signature would be a bump in the dijet invariant mass spectrum, as well as new interactions giving rise to special features in the dijet angular distributions. In fact, QCD calculations predict that high $-p_{\mathrm{T}}$ dijet production is dominated by $\mathrm{t}$-channel gluon exchange, leading to angular distributions that are peaked at $\left|\cos \theta^{*}\right|$ close to 1 , where $\theta^{*}$ is the polar scattering angle in the two-parton center-ofmass frame. By contrast, models of new processes characteristically predict angular distributions that would be more isotropic than those of QCD.

Many models of new physics predict the existence of additional heavy gauge bosons, where the charged ones are commonly denoted $W^{\prime}$. Such particles are most easily searched for in their decay to a charged lepton (either electron or muon) and a neutrino. 

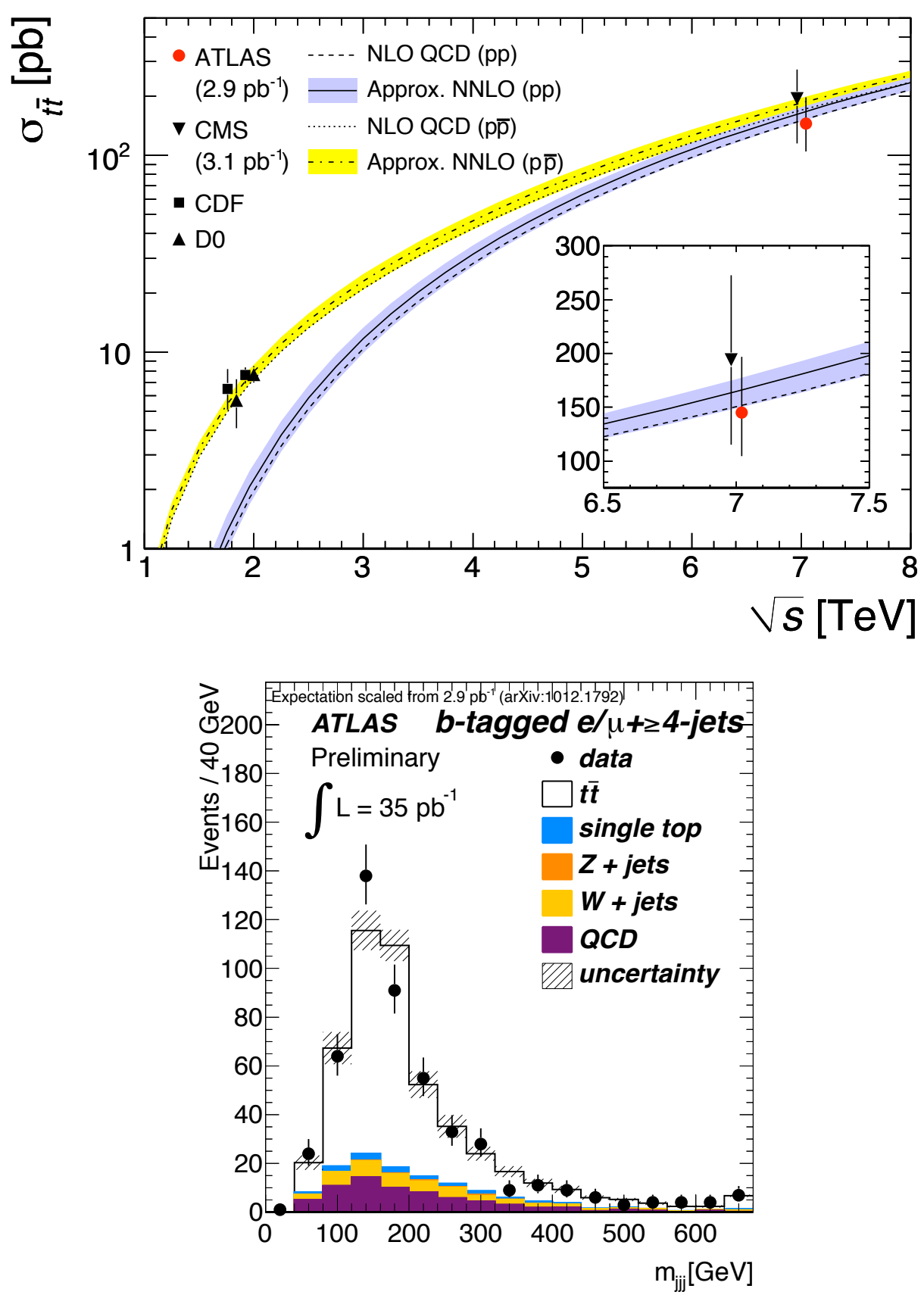

Figure 19: Top: $t \bar{t}$ production cross-section at hadron colliders as measured by CDF and D0 at Tevatron, CMS and ATLAS at LHC. The theoretical predictions for proton-proton and proton-antiproton collisions include the scale and PDF uncertainties and assume a top-quark mass of $172.5 \mathrm{GeV}$. Bottom: Invariant mass of the three jets with the largest combined $p_{\mathrm{T}}$ in semileptonic $t \bar{t}$ candidate events with at least one b-tagged jet. 


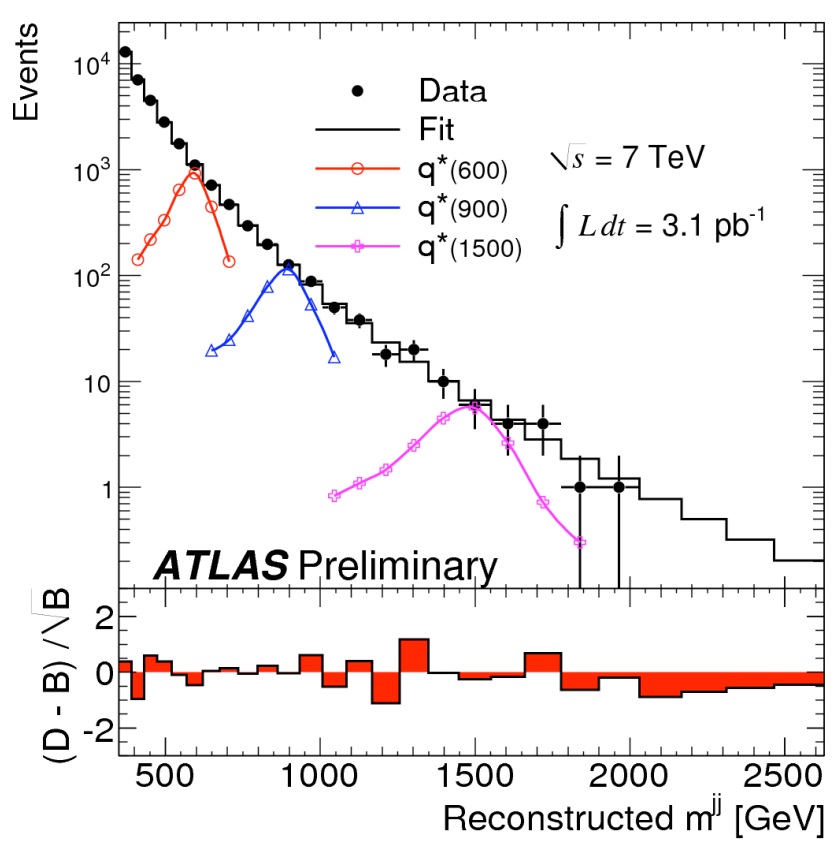

Figure 20: Dijet invariant mass distribution as measured in the data (filled points) and fitted using a binned background distribution (histogram). The predicted $q^{*}$ signals for excited-quark masses of 600, 900, and $1500 \mathrm{GeV}$ are overlaid. The bin-by-bin significance of the data-background difference is also shown.

\subsubsection{New Phenomena with Jets}

The measured distribution of the dijet invariant mass shown in figure 20, based on about one tenth of the available data, was used to set limits on the production of excited quarks $\left(q^{*}\right)$ as a function of the $q^{*}$ mass. Excited quarks have been excluded at the $95 \% \mathrm{CL}$ in the mass interval $0.30<m_{q^{*}}<1.53 \mathrm{TeV}$, extending the reach of previous experiments.

The distribution of the variable $\chi=\exp \left(\left|y_{1}-y_{2}\right|\right)$, where $y_{1,2}$ are the rapidities of the two jets emerging from " $2 \rightarrow 2$ " scattering processes, is predicted to be almost flat by QCD, whereas new processes tend to produce more events at low $\chi$. Moreover, new processes are expected to produce more central activity than QCD ones, so that their signal would appear as an increase in the ratio of the number of events in which the two highest $p_{\mathrm{T}}$ jets both fall into the central region to the number of events in which the two highest $p_{\mathrm{T}}$ jets both fall into the non-central region. By studying these variables, ATLAS excluded contact interactions up to a compositness scale of $3.4 \mathrm{TeV}$, again extending exclusion limits set at previous experiments. The measured distribution of the variable $\chi$ is shown in figure 21 .

\subsubsection{Search for $W^{\prime}$}

A first limit on the production of $W^{\prime}$ was set by assuming the same couplings as those of the Standard Model $W$ boson (Sequential Standard Model) and considering the $W^{\prime} \rightarrow e v$ decay channel. The measured distribution of the electron-missing energy transverse mass defined in section 


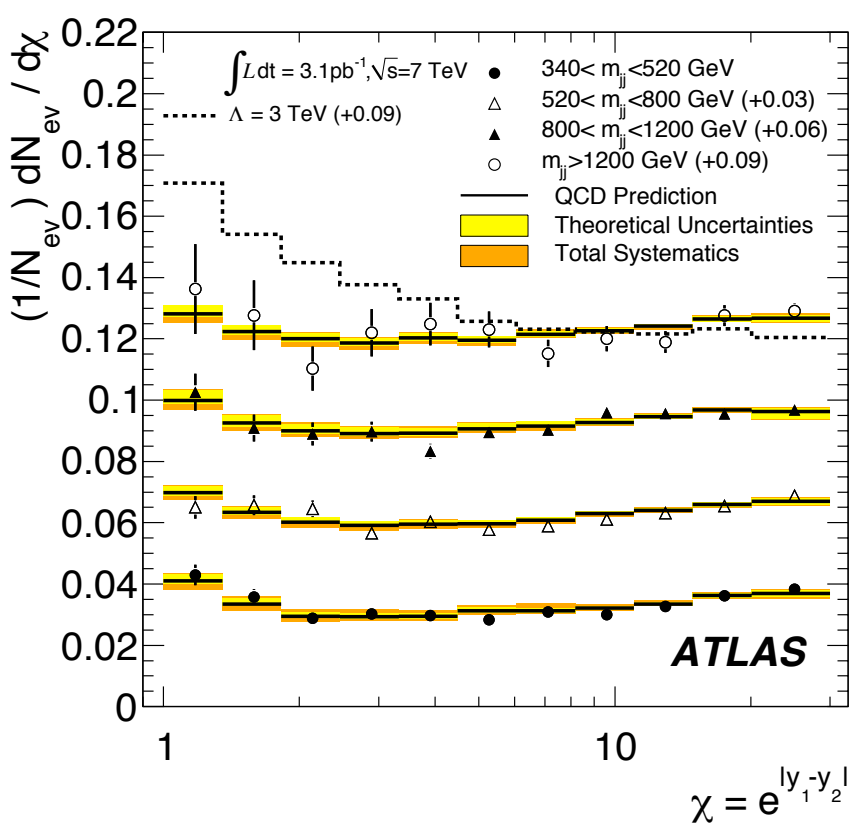

Figure 21: Normalized $\chi$ distributions for $340<m_{\mathrm{jj}}<520 \mathrm{GeV}, 520<m_{\mathrm{jj}}<800 \mathrm{GeV}$, and $800<m_{\mathrm{jj}}<$ $1200 \mathrm{GeV}$, with plotting offsets shown in parentheses. Shown are the QCD predictions with systematic uncertainties (bands), and data points with statistical uncertainties. The prediction for QCD with an added quark contact term with $\Lambda=3.0 \mathrm{TeV}$ is shown for the highest mass bin $m_{\mathrm{jj}}>1200 \mathrm{GeV}$.

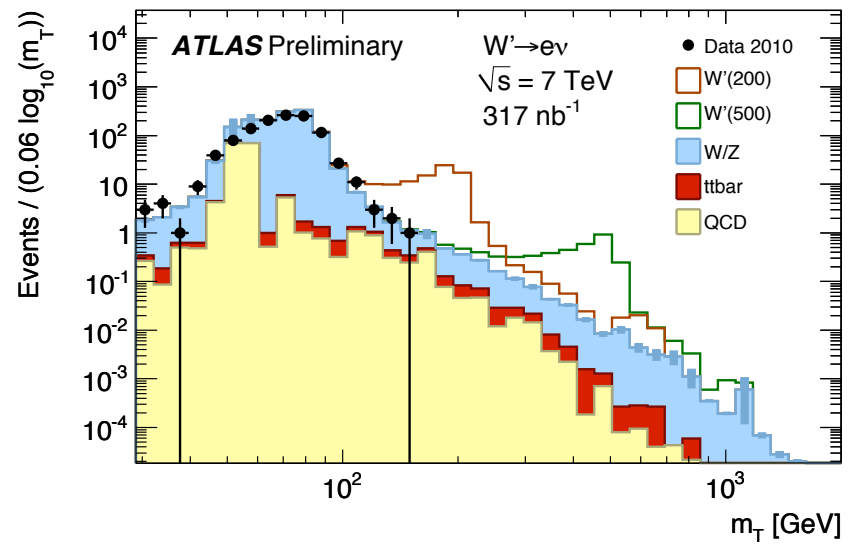

Figure 22: Spectrum of the electron-missing energy transverse mass. The points represent ATLAS data whereas the filled histograms show the Standard Model backgrounds. Open histograms are $W^{\prime}$ signals added to the background with masses as indicated in parentheses in the legend. The signal and other background samples are normalized to the data integrated luminosity. 


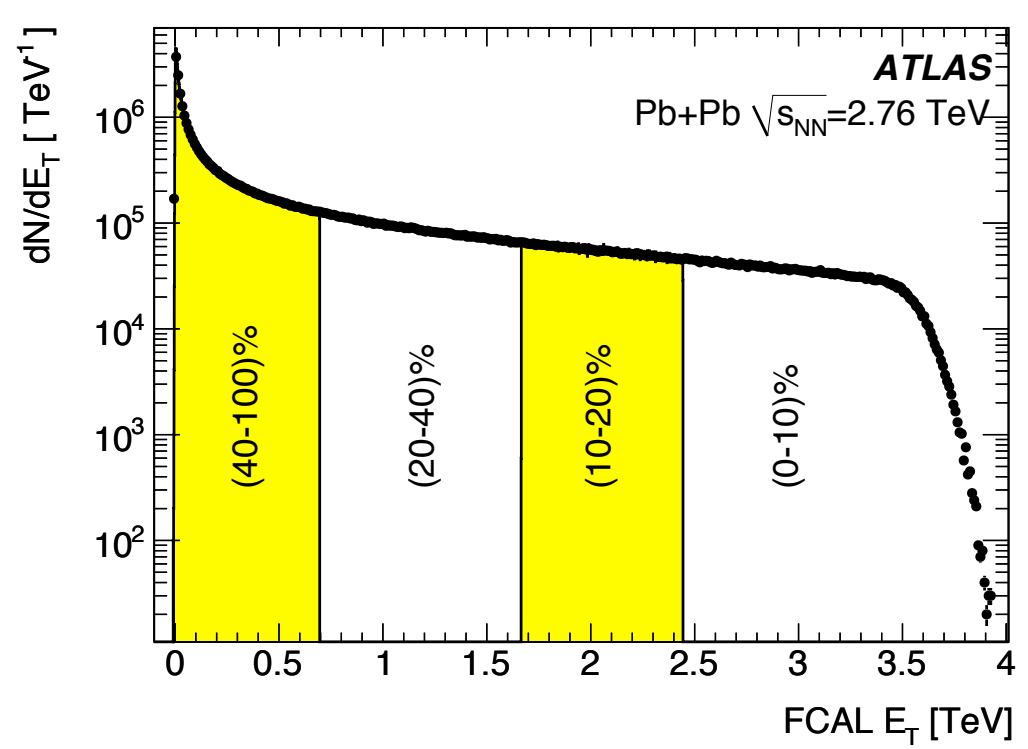

Figure 23: Distribution of uncorrected $\sum E_{\mathrm{T}}$ in the Forward Calorimeter (FCal). Bins in event activity or "centrality" are indicated by the alternating bands and labeled according to increasing fraction of lead-lead total cross section starting from the largest measured $\sum E_{\mathrm{T}}$.

4.3 is shown in figure 22 together with expected contributions from Standard Model processes. The Jacobian peaks expected for a $W^{\prime}$ with masses of 200 or $500 \mathrm{GeV}$ are also shown. Previous exclusion limits on $W^{\prime}$ production were extended to $465 \mathrm{GeV}$ with a limited data sample of $317 \mathrm{nb}^{-1}$ and by considering only one decay channel, which shows the wide discovery potential of LHC.

\section{Physics Results from Pb-ion Collisions}

Collisions of lead ions at ultra-relativistic energies are expected to produce a dense state with temperatures exceeding two trillion Kelvin. If deconfinement is indeed reached, the relevant degrees of freedom are not hadrons, but quarks and gluons. In this medium, high-energy quarks and gluons are expected to transfer energy to the medium by multiple interactions with the ambient plasma.

The study of events produced at ultra-relativistic heavy-ion collisions is typically carried out by labelling the events eccording to their geometry, or centrality: the larger the centrality, the larger the number of interacting nuclei and the event activity. At ATLAS, the level of event activity is characterized by using the total transverse energy $\left(\sum E_{\mathrm{T}}\right)$ deposited in the Forward Calorimeters as shown in figure 23. Bins are defined in centrality according to fractions of the total lead-lead cross-section selected by the trigger and are expressed in terms of percentiles $(0-10 \%, 10-20 \%$, 20-40\% and 40-100\%) with $0 \%$ representing the upper end of the $\sum E_{\mathrm{T}}$ distribution.

\subsection{Observation of Jet Quenching}

Looking for "jet quenching", the production of highly unbalanced dijets when one jet is pro- 

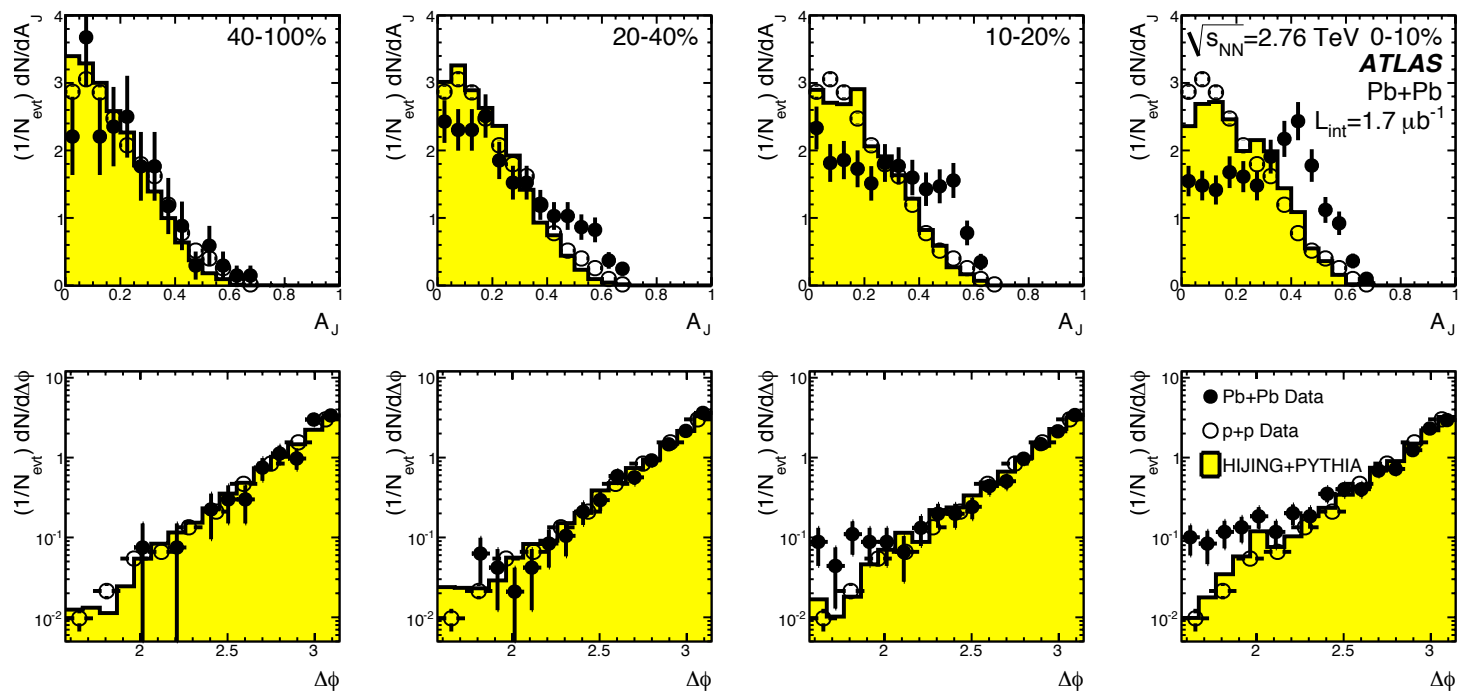

Figure 24: (top) Dijet asymmetry distributions for data (points) and unquenched HIJING with superimposed PYTHIA dijets (solid histograms), as a function of collision centrality (left to right from peripheral to central events). Proton-proton data from $\sqrt{s}=7 \mathrm{TeV}$, analyzed with the same jet selection, is shown as open circles. (bottom) Distribution of $\Delta \phi$, the azimuthal angle between the two jets, for data and HIJING+PYTHIA, again as a function of centrality.

duced at the periphery of the collision, has been proposed as a way to study in-medium QCD energy losses. Due to limited rapidity coverage and jet energies comparable to the underlying event energy, experiments at RHIC could not come to strong conclusions on this subject. Abundant production of jets with energies in excess of $100 \mathrm{GeV}$ is expected at LHC nucleon-nucleon centre-of-mass energies. In addition, the large acceptance and high granularity of the ATLAS calorimeters make it well suited for measuring jets. A very early study by ATLAS [24] was thus focussed on the balance between the highest transverse energy pair of jets in events where those jets have a sufficient azimuthal $(\phi)$ angle separation (opposite hemispheres). The jet energy imbalance was expressed in terms of the asymmetry $A_{J}$,

$$
A_{J}=\frac{E_{\mathrm{T} 1}-E_{\mathrm{T} 2}}{E_{\mathrm{T} 1}+E_{\mathrm{T} 2}}, \Delta \phi>\frac{\pi}{2}
$$

where the first jet was required to have a transverse energy $E_{\mathrm{T} 1}>100 \mathrm{GeV}$, and the second jet was the highest transverse energy jet in the opposite hemisphere with $E_{\mathrm{T} 2}>25 \mathrm{GeV}$. The average contribution of the underlying event energy was subtracted event-by-event, giving estimates of jet transverse energy above the ambient background. The transverse energies of dijets in opposite hemispheres were observed to become systematically more unbalanced with increasing event centrality leading to a large number of events which contain highly asymmetric dijets. The dijet asymmetry and $\Delta \phi$ distributions are shown in four centrality bins in figure 24 , where they are compared with proton-proton data and with fully-reconstructed HIJING[25]+PYTHIA simulated events. The simulated events are only intended to illustrate the effect of the $\mathrm{Pb}$ ion background on jet reconstruction. The dijet asymmetry in peripheral lead-lead events is similar to that in both 

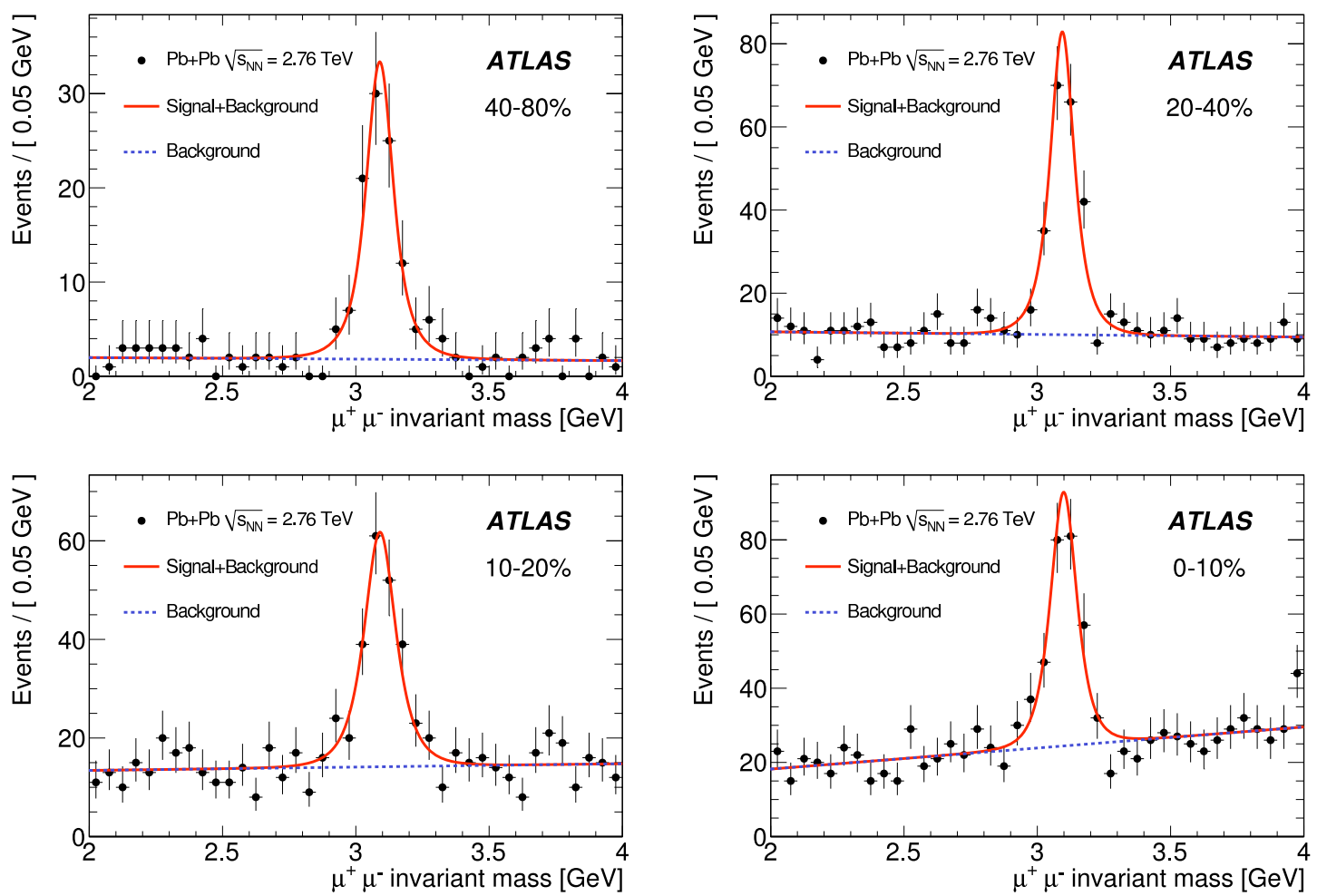

Figure 25: $\mu^{+} \mu^{-}$candidate invariant mass spectra in four centrality bins from most peripheral (40-80\%) to most central $(0-10 \%)$. The $J / \psi$ yields in each centrality bin are obtained using a sideband technique. The fits shown here are used as a cross check.

proton-proton and simulated events; however, as the events become more central, the lead-lead data distributions develop different characteristics, indicating an increased rate of highly asymmetric dijet events. This was the first observation of an enhancement of events with such large dijet asymmetries.

\section{2 $J / \psi$ Production Versus Event Centrality}

The measurement of quarkonia production in ultra-relativistic heavy ion collisions provides a potentially powerful tool for studying the formation of quark-gluon plasma. If deconfined matter is formed, then colour screening is expected to prevent the formation of quarkonium states when the screening length becomes shorter than the quarkonium size. However, data from previous experiments suggest that other mechanisms may come into play. Measurements at LHC energies, where higher temperatures and heavy quark production rates are expected, may address these debates with new experimental input. In addition, the production of $\mathrm{Z}$ bosons available at LHC energies can serve as a reference process for $J / \psi \rightarrow \mu^{+} \mu^{-}$production, since $Z$ bosons are not expected to be affected by the medium, although modifications to the nuclear parton distribution functions ought to be considered.

In a sample of minimum-bias lead-lead collisions corresponding to an integrated luminosity of $6.7 \mu \mathrm{b}^{-1}$, ATLAS reconstructed $J / \psi$ mesons via their decays to $\mu^{+} \mu^{-}$pairs in events char- 

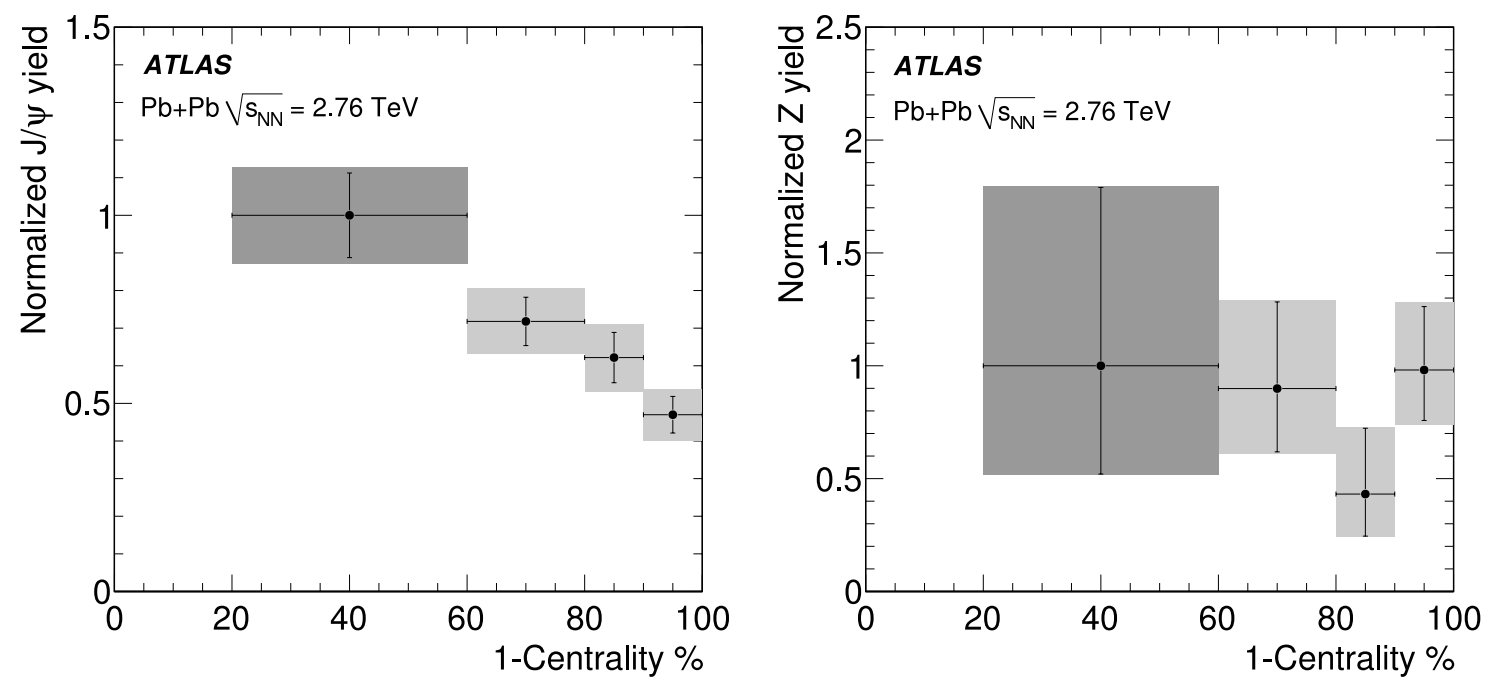

Figure 26: Normalized yeld of $J / \psi$ (left) and $Z$ boson (right), as described in the text, as a function of centrality. The statistical errors are shown as vertical bars while the grey boxes also include the combined systematic errors. The darker box indicates that the $40-80 \%$ bin is used to set the scale for all bins, but the uncertainties in this bin are not propagated into the more central ones.

acterized by different centralities. The corresponding peaks in the invariant mass distributions of $\mu^{+} \mu^{-}$candidates are shown in figure 25 . The measured yields are corrected by the reconstruction efficiency, derived from MC, and the width of the centrality bins. A "relative yield" is defined by normalizing to the yield found in the most peripheral 40-80\% centrality bin and, finally, a "normalized yield" is defined by scaling the relative yield by the ratio of the mean number of binary collisions in each centrality bin to that for the most peripheral (40-80\%) bin. The mean number of binary nucleon-nucleon collisions corresponding to each centrality bin was calculated using a Glauber Monte Carlo package that has been applied extensively at RHIC energies. The dependence of the normalized $J / \psi$ yield on the event centrality is shown in figure 26 , where a significant decrease from peripheral to central collisions can be observed, in qualitative agreement with previous, lower energy experiments. The relative yield of $Z$ as a function of centrality is also shown, although no conclusion can be inferred because of limited statistics: a total of $38 Z \rightarrow \mu^{+} \mu^{-}$candidates was selected in the $\mu^{+} \mu^{-}$mass window of 66 to $116 \mathrm{GeV}$.

\section{Conclusions}

2010 was an extremely exciting year for LHC. The accelerator reached all the announced goals, and the detectors performed equally well. ATLAS data collection was very smooth, and data have been under deep scrutiny to understand the details of the detector behaviour as well as tune detector-related effects in simulations.

Overall, 2010 was the year of Standard Model rediscovery, as is frequently said. No evidence for physics beyond the Standard Model has been found yet, although previous limits on the production of new particles start to be extended, even with limited statistics. A lot of new results 
are just about to come. Some model tuning is apparently needed for diffractive physics. Ultrarelativistic lead-ion collisions carried most of the surprises, with the first impressive evidence for jet-quenching. And we are just at the beginning of a very promising period to come.

\section{References}

[1] G. Aad et al., ATLAS Collaboration, The ATLAS Experiment at the Large Hdron Collider, JINST 3 (2008) S08003.

[2] LHC, L. Evans, (ed.), P. Bryant, (ed.), The CERN Large Hadron Collider: Accelerator and Experiments, JINST 3 (2008) S08001.

[3] M. Cacciari, G. P. Salam and G. Soyez, The anti-kt jet clustering algorithm, JHEP 04 (2008) 063.

[4] The ATLAS Collaboration, Jet energy scale and its systematic uncertainty in ATLAS for jets produced in proton-proton collisions at sqrts $=7 \mathrm{TeV}$, ATL-CONF-2010-058 (2010).

[5] S. van der Meer, Calibration of the effective beam height in the ISR, CERN-ISR-PO-68-31, 1968.

[6] G. Aad et al., ATLAS Collaboration, Luminosity Determination in pp Collisions at $\sqrt{s}=7 \mathrm{TeV}$ Using the ATLAS Detector at the LHC, accepted by EPJC, arXiv:1101.2185v1 [hep-ex].

[7] T. Sjostrand, S. Mrenna, P. Skands, PYTHIA 6.4 Physics and Manual, JHEP 0605 (2006) 026 [hep-ph/0603175].

[8] R. Engela, and J. Ranftb, Color singlet exchange between jets and the PHOJET Monte Carlo, Nuclear Phys. B - Proceedings Supplements Vol. 75, Issues 1-2 (1999), 272-274.

[9] G. Aad et al., ATLAS Collaboration, Charged-particle multiplicities in pp interactions measured with the ATLAS detector at the LHC, accepted by New J Phys, arXiv:1012.5104v2 [hep-ex].

[10] G. Aad et al., ATLAS Collaboration, Measurement of underlying event characteristics using charged particles in pp collisions at $\sqrt{s}=900 \mathrm{GeV}$ and $7 \mathrm{TeV}$ with the ATLAS detector, accepted by Phys. Rev. $D$, arXiv:1012.0791v2 [hep-ex].

[11] G. Aad et al., ATLAS Collaboration, Measurement of inclusive jet and dijet cross sections in proton-proton collisions at $7 \mathrm{TeV}$ centre-of-mass energy with the ATLAS detector, Eur. Phys. J. C71 (2011) 1512, arXiv:1009.5908v2 [hep-ex].

[12] G. Aad et al., ATLAS Collaboration, Study of Jet Shapes in Inclusive Jet Production in pp Collisions at $\sqrt{s}=7$ TeV using the ATLAS Detector, Phys. Rev. D 83, 052003 (2011), arXiv:1101.0070v1 [hep-ex].

[13] G. Aad et al., ATLAS Collaboration, Measurement of the $W \rightarrow \ell v$ and $Z / \gamma^{*} \rightarrow \ell^{+} \ell^{-}$production cross sections in proton-proton collisions at $\sqrt{s}=7 \mathrm{TeV}$ with the ATLAS detector, JHEP 12 (2010) 060, arXiv:1010.2130v1 [hep-ex].

[14] G. Aad et al., ATLAS Collaboration, Measurement of the production cross section for W-bosons in association with jets in pp collisions at $\sqrt{s}=7 \mathrm{TeV}$ with the ATLAS detector, accepted by Phys. Lett. $B$, arXiv:1012.5382v2 [hep-ex].

[15] M.L. Mangano, M. Moretti, F. Piccinini, R. Pittau, A. D. Polosa, ALPGEN, a generator for hard multiparton processes in hadronic collisions, JHEP 307 (2003) 001 [hep-ph/ 0206293 ].

[16] T. Gleisberg, S. Hoche, F. Krauss, M. Schonherr, S. Schumann, F. Siegert, J. Winter, Event generation with Sherpa 1.1, JHEP 02 (2009) 0072 
[17] J.M. Campbell, R.K. Ellis, D. Rainwater, Next-to-leading order QCD predictions for W+2 jet and Z+2 jet production at the CERN LHC, Phys. Rev. D 68 (2003) 094021 [hep-ph/ 0308195 ].

[18] G. Aad et al., ATLAS Collaboration, Measurement of the inclusive isolated prompt photon cross section in pp collisions at $\sqrt{s}=7$ TeV with the ATLAS detector, Phys. Rev. D 83, 052005 (2011), arXiv:1012.4389v2 [hep-ex] .

[19] G. Aad et al., ATLAS Collaboration, Measurement of the top quark-pair production cross section with ATLAS in pp collisions at $\sqrt{s}=7 \mathrm{TeV}$, accepted by EPJC, arXiv:1012.1792v2 [hep-ex].

[20] G. Aad et al., ATLAS Collaboration, Search for high-mass states with lepton plus missing transverse energy using the ATLAS detector with $36 \mathrm{pb}^{-1}$ of pp collisions at $\sqrt{\mathrm{s}}=7 \mathrm{TeV}$, submitted to Phys. Lett. $B$, arXiv:1103.1391v2 [hep-ex].

[21] G. Aad et al., ATLAS Collaboration, Search for Quark Contact Interactions in Dijet Angular Distributions in 7 TeV Proton-Proton Collisions with the ATLAS Detector at the LHC, Phys. Lett. B 694 (2011) 327-345, arXiv:1009.5069v1 [hep-ex].

[22] G. Aad et al., ATLAS Collaboration, Search for New Particles in Two-Jet Final States in 7 TeV Proton-Proton Collisions with the ATLAS Detector at the LHC, Phys. Rev. Lett. 105, 161801 (2010), arXiv:1008.2461v2 [hep-ex].

[23] G. Aad et al., ATLAS Collaboration, Search for New Physics in Dijet Mass and Angular Distributions in pp Collisions at $\sqrt{s}=7 \mathrm{TeV}$ Measured with the ATLAS Detector, submitted to New J Phys, arXiv:1103.3864v2 [hep-ex].

[24] G. Aad et al., ATLAS Collaboration, Observation of a centrality-dependent dijet asymmetry in lead-lead collisions at $\sqrt{s}_{\mathrm{NN}}=2.76 \mathrm{TeV}$ with the ATLAS detector at the LHC, Phys. Rev. Lett. 105, 252303 (2010), arXiv:1011.6182v2 [hep-ex].

[25] X.-N. Wang and M. Gyulassy, HIJING: A Monte Carlo model for multiple jet production in pp, $p A$, and AA collisions, Phys. Rev. D 44, 3501-3516 (1991)

[26] G. Aad et al., ATLAS Collaboration, Measurement of the centrality dependence of $J / \psi$ yields and observation of $Z$ production in lead-lead collisions with the ATLAS detector at the LHC, Phys. Lett. B 697 (2011) 294-312, arXiv:1012.5419v1 [hep-ex]. 